A UNITED STATES DEPARTMENT OF COMMERCE PUBLICATION
$A 11100989880$

NBS

PUBLICATIONS
NAT'L INST OF STANDARDS \& TECH R.I.C.

A11100989880

INBS monograph
OC100.U556 V136;1973 C.2 NBS-PUB-C 1958

\title{
Graphical
}

Recoupling of

U.S.

DEPARTMENT

OF

COMMERCE

National

Bureau

of

Standards

$-Q G$

100

.4556 no. 136

1973

c. 2

\section{Angular Momenta}


The National Bureau of Standards ${ }^{1}$ was established by an act of Congress March 3, 1901. The Bureau's overall goal is to strengthen and advance the Nation's science and technology and facilitate their effective application for public benefit. To this end, the Bureau conducts research and provides: (1) a basis for the Nation's physical measurement system, (2) scientific and technological services for industry and government, (3) a technical basis for equity in trade, and (4) technical services to promote public safety. The Bureau consists of the Institute for Basic Standards, the Institute for Materials Research, the Institute for Applied Technology, the Institute for Computer Sciences and Technology, and the Office for Information Programs.

THE INSTITUTE FOR BASIC STANDARDS provides the central basis within the United States of a complete and consistent system of physical measurement; coordinates that system with measurement systems of other nations; and furnishes essential services leading to accurate and uniform physical measurements throughout the Nation's scientific community, industry, and commerce. The Institute consists of a Center for Radiation Research, an Office of Measurement Services and the following divisions:

Applied Mathematics - Electricity - Mechanics - Heat - Optical Physics - Nuclear Sciences $^{2}$ - Applied Radiation ${ }^{2}$ - Quantum Electronics ${ }^{3}$ - Electromagnetics ${ }^{3}$ - Time and Frequency ${ }^{3}$ - Laboratory Astrophysics ${ }^{3}$ - Cryogenics ${ }^{3}$.

THE INSTITUTE FOR MATERIALS RESEARCH conducts materials research leading to improved methods of measurement, standards, and data on the properties of well-characterized materials needed by industry, commerce, educational institutions, and Government; provides advisory and research services to other Government agencies; and develops, produces, and distributes standard reference materials. The Institute consists of the Office of Standard Reference Materials and the following divisions:

Analytical Chemistry - Polymers - Metallurgy - Inorganic Materials - Reactor Radiation - Physical Chemistry.

THE INSTITUTE FOR APPLIED TECHNOLOGY provides technical services to promote the use of available technology and to facilitate technological innovation in industry and Government; cooperates with public and private organizations leading to the development of technological standards (including mandatory safety standards), codes and methods of test; and provides technical advice and services to Government agencies upon request. The Institute consists of a Center for Building Technology and the following divisions and offices:

Engineering and Product Standards - Weights and Measures — Invention and Innovation - Product Evaluation Technology - Electronic Technology - Technical Analysis - Measurement Engineering - Structures, Materials, and Life Safety * Building Environment ${ }^{4}$ - Technical Evaluation and Application ${ }^{4}$ - Fire Technology.

THE INSTITUTE FOR COMPUTER SCIENCES AND TECHNOLOGY conducts research and provides technical services designed to aid Government agencies in improving cost effectiveness in the conduct of their programs through the selection, acquisition, and effective utilization of automatic data processing equipment; and serves as the principal focus within the executive branch for the development of Federal standards for automatic data processing equipment, techniques, and computer languages. The Center consists of the following offices and divisions:

Information Processing Standards - Computer Information - Computer Services Systems Development — Information Processing Technology.

THE OFFICE FOR INFORMATION PROGRAMS promotes optimum dissemination and accessibility of scientific information generated within NBS and other agencies of the Federal Government; promotes the development of the National Standard Reference Data System and a system of information analysis centers dealing with the broader aspects of the National Measurement System; provides appropriate services to ensure that the NBS staff has optimum accessibility to the scientific information of the world. The Office consists of the following organizational units:

Office of Standard Reference Data - Office of Technical Information and Publications Library - Office of International Relations.

\footnotetext{
1 Headquarters and Laboratories at Gaithersburg, Maryland, unless otherwise noted; mailing address Washington, D.C. 20234.

3 Part of the Center for Radiation Research.

3 Located at Boulder, Colorado 80302.

- Part of the Center for Building Technology.
} 


\section{Graphical Recoupling of Angular Momenta}

National Bureau oí stantre:ds

APR 291974

D. R. Lehman

Department of Physics

The George Washington University

Washington, D.C. 20006

and

\section{J. S. O’Connell}

Center for Radiation Research Institute for Basic Standards

National Bureau of Standards

Washington, D.C. 20234

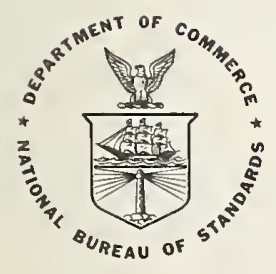

U.S. DEPARTMENT OF COMMERCE, Frederick B. Dent, Secretary

NATIONAL BUREAU OF STANDARDS, Richard W. Roberts, Director

Issued October 1973 
Library of Congress Catalog Number: 73-600149

\section{National Bureau of Standards Monograph 136}

Nat. Bur. Stand. (U.S.), Monogr. 136, 18 pages (Oct. 1973)

CODEN: NBSMA6

For sale by the Superintendent of Documents, U.S. Government Printing Office, Washington, D.C. 20402 (Order by SD Catalog No. C13.44:136)

Price 50 cents

Stock Number 0303-01160 


\title{
Graphical Recoupling of Angular Momenta
}

\author{
D. R. Lehman* and J. S. O'Connell
}

\begin{abstract}
A diagrammatic method for solving angular momentum recoupling problems is presented. It is shown that a few graphical elements with a set of rules for their use lead to the solution of many types of recoupling problems in an intuitive and systematic way. Several examples are given together with exercises to develop the reader's facility with the method.
\end{abstract}

Key words: Angular momentum; diagrams; graphs; quantum theory; recoupling; transformation theory.

\section{Introduction}

Many calculations in atomic, nuclear, and elementary particle physics involve spin-angular integrals where the integrand is the product of three or more functions that possess definite angular momentum. They arise, for example, in the computation of matrix elements of the multipole operator with many-electron wave functions in atomic physics and in the determination of angular distributions in photonuclear physics. The evaluation of such integrals by application of angular momentum recoupling algebra tends to be a tedious task. Recently, however, M. Danos [1] ${ }^{1}$ has presented a graphical method whereby this complex process can be carried through in a transparent way. Danos introduces a phase convention which eliminates minus signs from the interior part of the calculation, thus greatly reducing the chance of computational error. This graphical method resembles that of constructing a flowchart before writing a computer program, and it is an excellent bookkeeping technique for recoupling algebra, just as Feynman's diagrams are in quantum electrodynamics.

Other diagrammatic methods [2, 3] have been developed as aids to angular momentum calculations. The reader can find additional references to the literature in [2]. These methods usually start by assigning a graphical symbol to the vector-coupling coeff. cient (3-j or Clebsch Gordan) and compound the vector coupling to the $6-j$ or $9-j$ coefficients. Danos' approach focuses on the recoupling aspect of angular momentum theory and uses four-element recoupling as its basic symbol. With the $9-j$ recoupling as the central graphical element, separate graphical elements need not be introduced for the lower symbols since they are already included at no loss of

\footnotetext{
*National Research Council Postdoctoral Research Associate. Present Address: Department of Physics, The George W ashington University. Washington, D.C. 20006.

'Firures in brackets indicate the literature references at the end of this paper.
}

simplicity. For example, the $6-j$ recoupling is a $9-j$ recoupling with one of the angular momenta equal to zero. Moreover, the judicious choice of phase convention for the contrastandard elements, given in eq (1), removes the distinction between states and operators, thus eliminating phases during the recoupling. We feel these features give the method a power and elegance for recoupling problems not found in the previous schemes.

Our objective is to present a didactic exposition of Danos' diagrammatic method for angular momen. tum recoupling algebra which will be useful for classroom use or self-learning. The reader or student is assumed to have a rudimentary knowledge of angular momentum theory comparable to that obtained in a graduate quantum mechanics course at the level of Merzbacher [4], Messiah [5], or Schiff [6]. The material discussed below is equivalent to or parallels that in Edmond's monograph [7]. In section 2, we introduce the fundamental elements of the diagrammatic method and the rules for their application. Symbols are introduced for coupled elements, normalization, insertion of a complete set of states, projection integrals, the contraction of two integral angular momenta, grouping of momenta, and the recoupling of two pairs of coupled momenta. The tools developed in section 2 are then applied in section 3 to simple examples from the different types of problems to which the method is applicable. These include the computation of the multipole operator matrix element for single-particle states in the $j$ representation, the derivation of a sum rule for the 6-j symbol, and the derivation of a general formula for a $(\gamma, n)$ angular distribution. The main text then closes in section 4 with several exercises, plus an. swers, by means of which the reader can develop facility with the method and experience the fun involved in its application. A series of appendices gives useful formulas and background material on the basis of the method. 


\section{Elements and Rules}

We first introduce the rotational wave functions and discuss their conjugation, coupling, normalization, and closure properties. Spherical harmonics are universally chosen to represent the rotational properties of wave functions and operators in physical systems possessing spherical symmetry and integral angular momenta. Danos has shown that multiplying the ordinary spherical harmonics, as defined by Edmonds [7], by a phase factor $(-i)^{l}$ eliminates a mismatch between certain properties of vector algebra and angular momentum coupling theory. Therefore, the rotational part of a wave function for integral angular momentum is defined as

$$
Y_{m}^{[\eta]}(\theta, \varphi)=(-i)^{l} Y_{l m}(\theta, \varphi) .
$$

Rotation functions with square bracketed superscripts are called contrastandard. Their Hermitian conjugate (or costandard) forms are given by

$$
Y_{m}^{[l]]^{\dagger}}=\tilde{Y}_{m}^{[l] C}=(-1)^{l+m} \tilde{Y}_{\underline{m}}^{[l]}=(-1)^{l+m} Y_{\underline{m}}^{[l]},
$$

where the tilde means transposition (which has no effect on spherical harmonics) and $C$ means complex conjugation. The two component spin- $-\frac{1}{2}$ wave function will be designated $\chi_{h_{s}}^{[1 / 2]}$. These functions and their representations are discussed in appendix $B$.

A general spin-angle wave function for a particle (with either integer or half-integer angular momentum) will be written as

$$
\left.\psi_{m}^{[j]}(\Omega) \text { with } \psi_{m}^{[j]}\right]^{\dagger}=(-1)^{j+m} \psi_{-m}^{[j]} .
$$

The coupling of the angular momenta of two particles to a total angular momentum $J$ is given by

$$
\begin{gathered}
\Psi_{M}^{[J]}\left(\Omega_{12}\right)=\sum_{m_{1} m_{2}}\left\langle j_{1} m_{1} j_{2} m_{2} \mid J M\right\rangle \psi_{m_{1}}^{\left[j_{1}\right]}\left(\Omega_{1}\right) \varphi_{m_{2}}^{\left[j_{2}\right]}\left(\Omega_{2}\right) \\
\equiv\left[\psi^{\left[j_{1}\right]}\left(\Omega_{1}\right) \times \varphi^{\left[j_{2}\right]}\left(\Omega_{2}\right)\right]_{M}^{[J]},
\end{gathered}
$$

and this equation is to be read as $j_{1}$ coupled with $j_{2}$ to $J$. The resultant $J$ can take on values from $\left|j_{1}-j_{2}\right|$ to $j_{1}+j_{2} .\left\langle j_{1} m_{1} j_{2} m_{2} \mid J M\right\rangle$ is a vector-coupling coefficient [7]. This coupling is represented graphically in figure 1 .

A common single-particle coupling is that of a particle's orbital and spin momenta

$$
\begin{aligned}
\psi_{m}^{[j]}=\left.\sum_{m_{l} m_{s}}\left\langle l m_{l} \frac{1}{2} m_{s} \mid j m\right\rangle Y\right|_{m l} ^{l} \chi_{m_{s}}^{[1 / 2]} & \\
& \equiv\left[Y^{[l]} \times \chi^{[1 / 2]}\right]_{m}^{[j]} .
\end{aligned}
$$

The normalization condition for wave functions like those in eqs (4) and (5) is written

$$
\int \psi_{m}^{[j] \dagger} \psi_{m}^{[j]}=1
$$
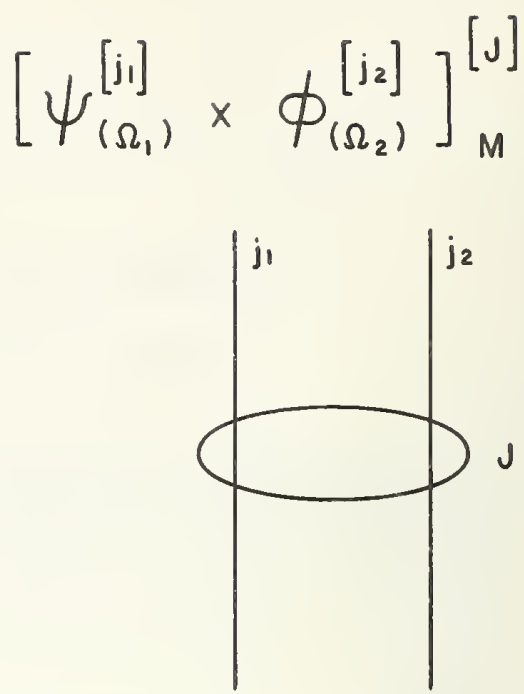

Figure 1. Coupled angular elements.

where $\int$ is defined to mean that all spin sums and angular integrations are to be performed. The product of wave functions in eq (6) can be expanded in terms of all possible coupled pairs

$$
\psi_{m}^{[j] \dagger} \psi_{m}^{[j]}=(-1)^{j+m} \sum_{J}\langle j-m j m \mid J 0\rangle\left[\tilde{\psi^{[j]}} \times \psi^{[j]}\right]_{0}^{[J]},
$$

since the vector-coupling coefficients satisfy

$$
\sum_{J}\langle j-m j m \mid J 0\rangle\left\langle j-m^{\prime} j m^{\prime} \mid J 0\right\rangle=\delta_{m m^{\prime}} .
$$

If $\psi^{[j]}$ were an ordinary spherical harmonic, the coupled pair in eq (7) could be replaced by a single spherical harmonic of angular momentum $J$ through use of the addition theorem for spherical'harmonics [7]. After integration over the angular coordinates, only the $J=0$ term survives, because

$$
\int Y_{0}^{[J]}(\Omega) d \Omega=\sqrt{4 \pi} \int Y_{0}^{[0]} Y_{0}^{[J]} d \Omega=\sqrt{4 \pi} \delta_{0 J} .
$$

Likewise, only the $J=0$ term survives the spin-angle integration when eq (7) is substituted into eq (6). This leads to

$$
\int\left[\tilde{\psi}[j] \times \psi^{[j]}\right]_{0}^{[0]}=(2 j+1)^{1 / 2} \equiv \hat{j}
$$

where we have used $\langle j-m j m \mid 00\rangle=(-1)^{j+m} / \hat{j}$. Equation (8) is the contrastandard element's analog of the normalization equation. A spin-angle integral over a zero coupling will be called a "projection integral," and will be represented graphically by a box as shown in figure 2a (also see appendix A). Thus, by eq (8), two lines terminating in a box gives the factor $\hat{j}$. 


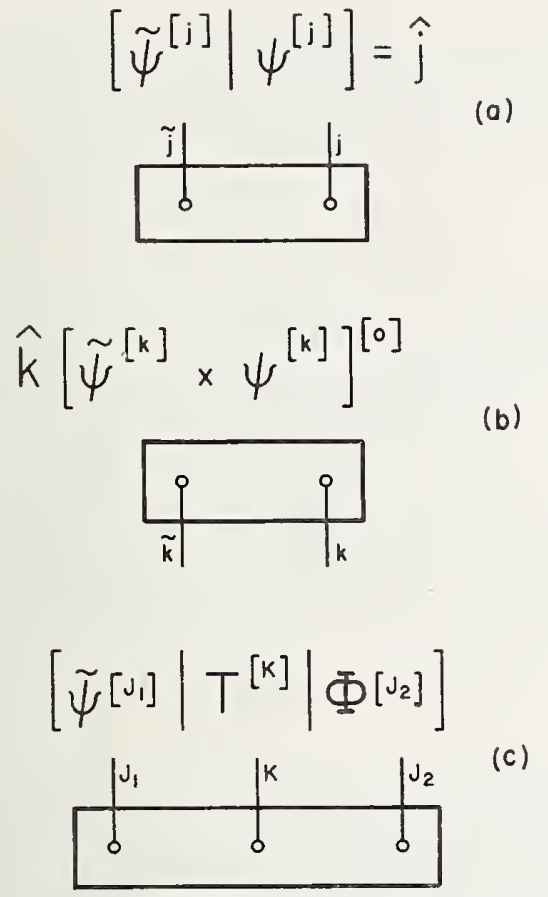

FIGURE 2. (a) Two-element projection integral; (b) insertion of intermediate states; $(c)$ projection integral of the invariant triple product.

Similarly, two lines taken out of a box, as in figure $2 \mathrm{~b}$, is the function $\hat{k}\left[\tilde{\psi}^{[k]} \times \psi^{[k]}\right]^{[0]}$. This function is useful as part of the representation for inserting a complete set of states into a recoupling problem by means of the closure relation for orthonormal functions. For integral momenta, the closure relation is

$$
\sum_{l m} Y_{m}^{[l]}(\Omega) Y_{m}^{[l]^{\dagger}}\left(\Omega^{\prime}\right)=\delta\left(\Omega-\Omega^{\prime}\right)
$$

In order to write eq (9) in the form of an angular momentum coupling, we use

$$
\begin{aligned}
{\left[Y^{[l]} \times Y^{[l]}\right]^{[0]}=\sum_{m}\langle l-m l m \mid 00\rangle Y_{-m}^{[l]} Y_{m}^{[l]} } \\
=\frac{1}{\hat{l}} \sum_{m} Y_{m}^{[l] Y_{m}^{[l] \dagger},}
\end{aligned}
$$

then eq (9) can be written as

$$
\sum_{l} \hat{l}\left[Y^{[l]}(\Omega) \times Y^{[l]}\left(\Omega^{\prime}\right)\right]^{[0]}=\delta\left(\Omega-\Omega^{\prime}\right) .
$$

Similarly, for the spin function

$$
\sum_{m_{s}} \chi_{m_{s}}^{[1 / 2]} \chi_{m_{s}}^{[1 / 2]^{\dagger}}=\left(\begin{array}{ll}
1 & 0 \\
0 & 1
\end{array}\right) \equiv \mathrm{I}
$$

which becomes

$$
-\frac{\hat{1}}{2}\left[\chi^{[1 / 2]} \times \tilde{\chi}^{[1 / 2]}\right]^{[0]}=\mathrm{I}
$$

In general, the closure relation for constrastandard elements is

$$
\sum_{k \alpha}(-1)^{2 k \hat{k}}\left[\psi_{\alpha}^{[k]} \times \tilde{\psi}_{\alpha}^{[k]}\right]^{[0]}=\mathfrak{\}},
$$

where $\alpha$ represents all other quantum numbers, e.g., radial, and $I$ is the unity operator in the space of the functions. For the usual spin-angle functions of eq (5), the unity operator is $\delta\left(\Omega-\Omega^{\prime}\right) \mathrm{I}$.

A graph for eq (14) can be constructed by using figure $2 \mathrm{~b}$ followed by a recoupling of the lines to move the element with the tilde to the right. This recoupling (as will be shown) introduces the required phase $(-1)^{2 k}$. The summation is automatic in any diagrammatic element that introduces new quantum numbers.

We next consider the coupling that arises in the evaluation of the matrix element of an operator $T$ between final and initial quantum states. If the states and operator have been expanded in terms of angular momentum eigenfunctions, the general matrix element will be of the form (see appendix A)

$$
\int\left[\tilde{\psi}^{\left[J_{1}\right]} \times\left[T^{[K]} \times \Phi^{\left[J_{2}\right]}\right]^{\left[J_{1}\right]}\right]^{[0]} .
$$

Note that, just as in the normalization integral, the total coupling is to zero since any other coupling will vanish in the integration. The internal brackets may be removed because the net result will be the same no matter which pair of functions is coupled first. The integral over the zero-coupled triple product is a projection integral and since it occurs frequently is given a special notation

$$
\left[\tilde{\psi}^{\left[J_{1}\right]}\left|T^{[K]}\right| \Phi^{\left[J_{2}\right]}\right] \equiv \int\left[\tilde{\psi}^{\left[J_{1}\right]} \times T^{[K]} \times \Phi^{\left[J_{2}\right]}\right]^{[0]} .
$$

Its graphical representation is shown in figure 2c. The projection integral of a triple product is closely related to the usual reduced matrix element (see appendix A),

$$
\left\langle\psi^{\left[J_{1}\right]} \| T^{[K]}|| \Phi^{\left[J_{2}\right]}\right\rangle=(-1)^{J_{1}+K^{\prime}-J_{2}}\left[\tilde{\psi}^{\left[J_{1}\right]}\left|T^{\left[K^{\prime}\right]}\right| \Phi^{\left[J_{2}\right]}\right] .
$$

Two coupled integral momenta of the same argument can be contracted by the formula

$$
\left[Y^{\left[l_{1}\right]}(\Omega) \times Y^{\left[l_{2}\right]}(\Omega)\right]^{[l]}=Q_{l_{1} l_{2}}^{[l]} Y^{[l]}(\Omega),
$$



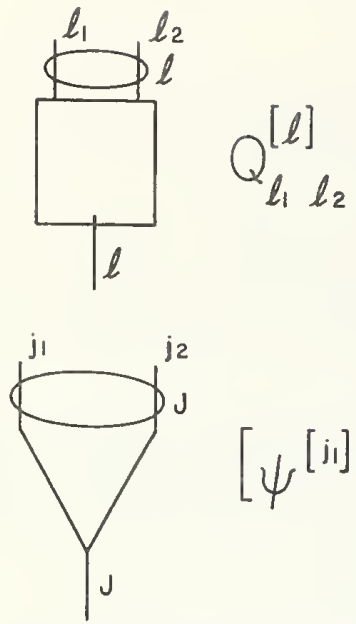

$$
\left[\psi^{\left[i_{1}\right]} \times \phi^{\left[j_{2}\right]}\right]^{[j]}
$$

FIGURE 3. (a) Contraction with a Q-box, (b) temporary grouping.

where

$$
Q_{l_{1} l_{2}}^{[l]}=(-1)^{\left(l_{1}+l_{2}+l\right) / 2} \frac{\hat{l}_{1} \hat{l}_{2}}{(4 \pi)^{1 / 2}}\left(\begin{array}{lll}
l_{1} & l_{2} & l \\
0 & 0 & 0
\end{array}\right),
$$

which we recognize as the addition theorem for spherical harmonics [7]. $\left(\begin{array}{lll}l_{1} & l_{2} & l_{3} \\ m_{1} & m_{2} & m_{3}\end{array}\right)$ is the Wigner 3-j symbol.

The graphical symbol, figure $3 \mathrm{a}$, called a $Q$-box, is used to represent this operation. On the other hand, any two coupled functions can be temporarily labeled by a single line, as in figure $3 \mathrm{~b}$. This is just a convenience and the two functions must eventually re-emerge from the grouping in the same order as they entered.

Finally, we consider the fundamental recoupling operation upon which the present system is based. All recouplings will be expressed as combinations of the two-pair recoupling transformation

$$
\begin{aligned}
& {\left[\left[\varphi^{[a]} \times \varphi^{[b]}\right]^{[c]} \times\left[\varphi^{[d]} \times \varphi^{[e]}\right]^{[f]}\right]^{[i]}} \\
& \quad=\sum_{g h}\left[\begin{array}{lll}
a & b & c \\
d & e & f \\
g & h & i
\end{array}\right]\left[\left[\varphi^{[a]} \times \varphi^{[d]}\right]^{[g]} \times\left[\varphi^{[b]} \times \varphi^{[e]}\right]^{[h]}\right]^{[i]}
\end{aligned}
$$

where the boxed coefficient is related to the usual 9-j coefficient by

$$
\left[\begin{array}{lll}
a & b & c \\
d & e & f \\
g & h & i
\end{array}\right]=\hat{c} \hat{f} \hat{g} \hat{h}\left\{\begin{array}{lll}
a & b & c \\
d & e & f \\
g & h & i
\end{array}\right\}
$$

with $\hat{j} \equiv(2 j+1)^{1 / 2}$. Equation (18) is expressed in words as follows: A system of four wave functions

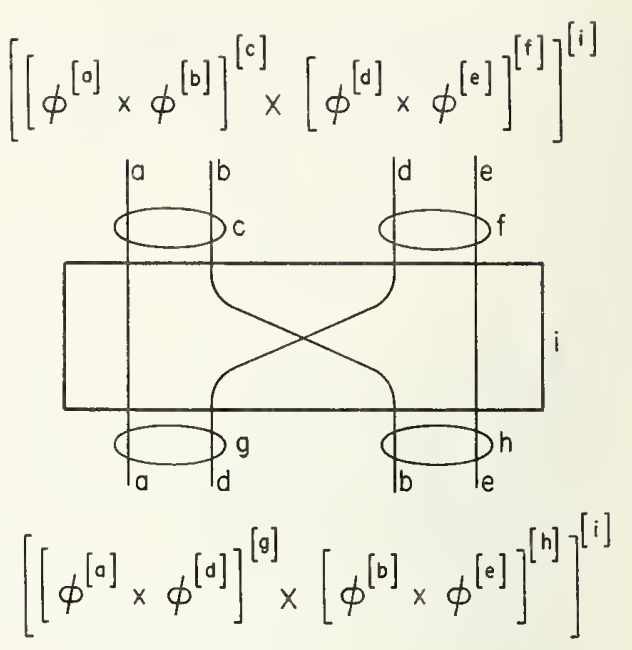

FiguRE 4. Two-pair recoupling box.

with angular momenta $a, b, d, e$ is originally coupled $a b$ to $c$, and $d e$ to $f$. The intermediate pair momenta $c$ and $f$ are coupled to the total system momentum $i$. If the pairs are recoupled $a d$ to $g$, and be to $h$, the original system wave function can be expanded in terms of the new system wave functions with intermediate momenta $g$ and $h$. The expansion coefficients are the boxed coefficients. The recoupling transformation is represented graphically by the diagram in figure 4 . Note the correspondence between the labeling of the line elements and loops in figure 4 and the boxed coefficient. The diagram is to be read from top to bottom. The values of $a$ through $f$ and $i$ are given; the intermediate momenta $g$ and $h$ can take on the range of values

$$
\begin{aligned}
& |a-d| \leqslant g \leqslant a+d \\
& |b-e| \leqslant h \leqslant b+e
\end{aligned}
$$

subject to $g, h$, and $i$ satisf ying triangularity.

Now we have sufficient tools to solve a variety of angular momentum recoupling problems. Symbols have been assigned to coupled elements (fig. 1), normalization (fig. 2a), insertion of states (fig. 2b), projection integrals (fig. 2c), the contraction of two integral angular momenta (fig. 3a), grouping of momenta (fig. $3 \mathrm{~b}$ ). and the recoupling of two-pairs of coupled momenta (fig. 4). These graphical elements are to be combined according to the following rules in problem solving:

Rule 1. Only coupled elements can be recoupled.

Rule 2. Lines are assigned to each distinct element in a coupled expression preserving the order.

Rule 3. Lines can cross only in a recoupling box as shown in figure 4. Mock zeros (dashed lines) can be added anytime to achieve four lines in a box. 
Rule 4. The initial coupling equals the final coupling times the product of all recoupling boxes and $Q$-boxes summed over any unspecified intermediate quantum numbers.

\section{Examples and Applications}

\subsection{The Projection Integral for Three Inte- gral Angular Momenta}

A physical problem in which the initial and final state wave functions and the transition operators are expanded in spherical harmonics leads to the projection integral

$$
\left[Y^{\left[l_{1}\right]}\left|Y^{\left[l_{2}\right]}\right| Y^{\left[l_{3}\right]}\right]=\int d \Omega\left[Y^{\left[l_{1}\right]} \times Y^{\left[l_{2}\right]} \times Y^{\left[l_{3}\right]}\right]^{[0]}
$$

The invariant triple product can be evaluated by the successive contraction of angular elements by $Q$-boxes as sliown in figure 5 . The first $Q l_{1}^{13} l_{2}$ replaces $\left[Y^{[/ 1]} \times Y^{[/ 2]}\right]^{[/ 3]}$ by $Y^{[/ 3]}$. The second contracts the two $Y^{\left[{ }^{13}\right]}$ 's to $Y^{[0]}$. Therefore, we have

$$
\begin{gathered}
{\left[Y^{\left[l_{1}\right]}\left|Y^{[/ 2]}\right| Y^{\left[l_{3}\right]}\right]=Q Q_{l_{1} l_{2}}^{\left[l_{3}\right]} Q_{l_{3} l_{3}}^{[0]} \int d \Omega Y^{[0]}} \\
=(-1)^{\left(l_{1}+l_{2}+l_{3} / 2\right.} \frac{\hat{l}_{1} \hat{l}_{2} \hat{l}_{3}}{(4 \pi)^{1 / 2}}\left(\begin{array}{ccc}
l_{1} & l_{2} & l_{3} \\
0 & 0 & 0
\end{array}\right) .
\end{gathered}
$$

This result will be very useful in what follows.

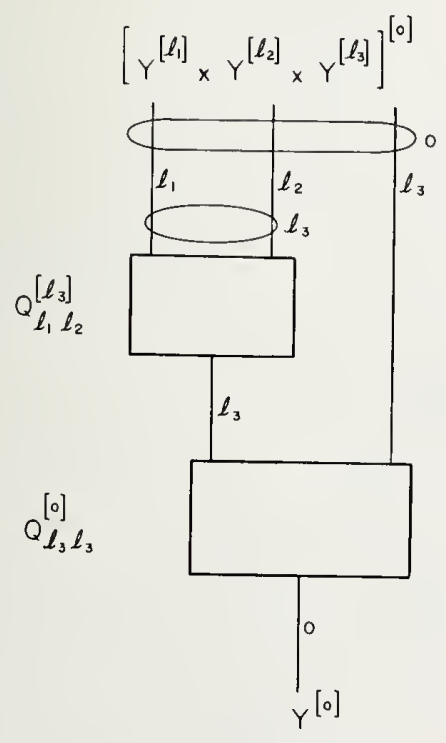

Figure 5. Two contractions.

\subsection{A Multipole Operator in $j$-Representation}

The expansion of the plane wave in angular elements

$$
e^{i q \cdot r}=(4 \pi)^{1 / 2} \sum_{L}(-1)^{L} \hat{L}_{j} j_{L}(q r) Y_{0}^{[L]}\left(\Omega_{r}\right)
$$

where $j_{l}$. is a spherical Bessel function and the direction of $\mathbf{q}$ is taken along the $z$-axis, gives the often encountered projection integral of the multipole operator $j_{L} Y^{[L]}$. For single particle states of eq (5), the Coulomb and other particle momentum and spin-independent operators have projection integrals of the form

$$
\begin{aligned}
& M_{L}(q) \equiv\left[R _ { l ^ { \prime } } ( r ) \left[Y^{\left[l^{\prime}\right]}\right.\right. \\
& \left.\left.\quad \times \tilde{\chi}^{[1 / 2]}\right]^{\left[j^{\prime}\right]}\left|j_{L}(q r) Y^{[L]}\right| R_{l}(r)\left[Y^{[l]} \times \chi^{[1 / 2]}\right]^{[j]}\right],
\end{aligned}
$$

where $R_{l}(r)$ are the radial functions with their radial quantum number, $n$, suppressed. Figure 6 shows the graphical recoupling involved to separate the radial, angular, and spin integrals. The box at the top of the figure is the projection integral to be evaluated (Rule 1); those at the bottom are standard projection integrals whose values we know. The recoupling boxes between them relate the two. Starting at the top, lines are assigned to each distinct element (Rule 2); in this case, the two orbital momenta $l$ and $l^{\prime}$, the two spin- $\frac{1}{2}$ functions,

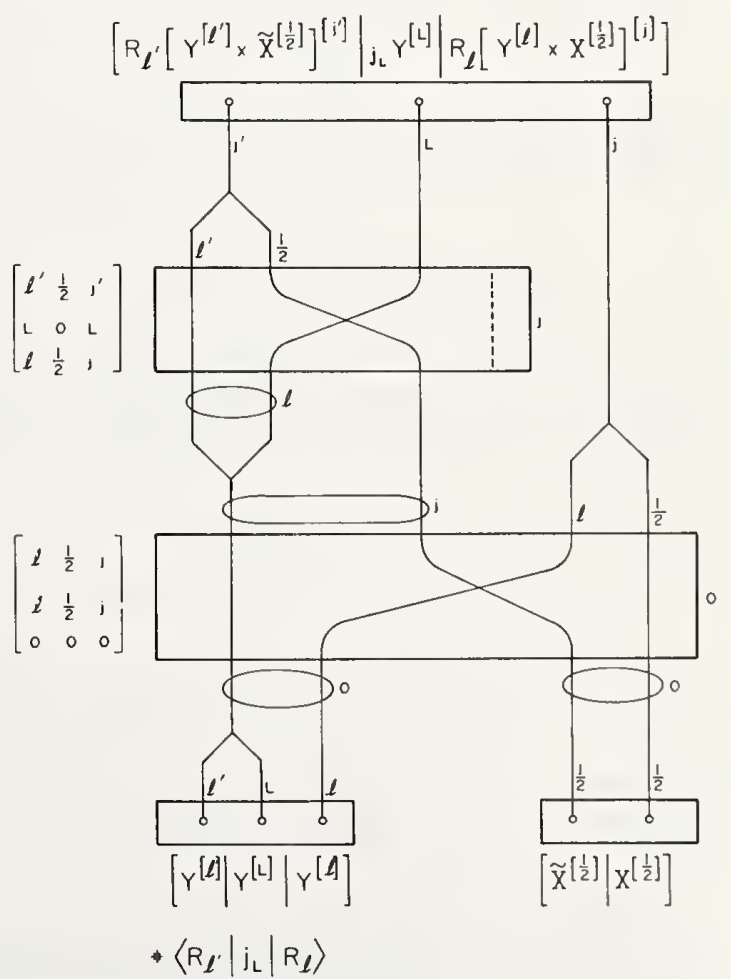

FIGURE 6. Flowchart for evaluating a multipole operator. 
and the operator of rank $L$. The first recoupling separates the spin function from the orbital function in the final (primed) state. A zero angular momentum (the dashed line) is added to make up the standard box (Rule 3). The fact that $L$ and $l^{\prime}$ couple to $l$ is not obvious at this point. The whole box has angular momentum $j$ since the total problem is coupled to 0 and the pair of lines excluded from the first box are coupled to $j$. The second box uncouples the orbital and spin functions of the initial (unprimed) state and couples all the spatial functions together and all the spin functions together. The final two loops must be zero because projection integrals are made over the final products. The invariant triple product among the $l, l^{\prime}$, and $L$ now show that $L$ and $l^{\prime}$ must be coupled to $l$ in the intermediate loop between boxes. In general, after a diagram is drawn, one looks at any apparently arbitrary intermediate couplings to see if they can in fact be specified by relationships elsewhere in the diagram. By Rule 4 then

$$
\begin{aligned}
& M_{L}(q)=\left[\begin{array}{lll}
l^{\prime} & \frac{1}{2} & j^{\prime} \\
L & 0 & L \\
l & \frac{1}{2} & j
\end{array}\right]\left[\begin{array}{lll}
l & \frac{1}{2} & j \\
l & \frac{1}{2} & j \\
0 & 0 & 0
\end{array}\right] \\
& {\left[\tilde{\chi}^{[1 / 2]} \mid \chi^{[1 / 2]}\right]\left[Y^{\left[l^{\prime}\right]}\left|Y^{[L]}\right| Y^{[l]}\right]\left\langle R_{l^{\prime}}\left|j_{L}(q r)\right| R_{l}\right\rangle .}
\end{aligned}
$$

The radial factor is the integral

$$
\left\langle R_{l^{\prime}}\left|j_{L}(q r)\right| R_{l}\right\rangle=\int_{0}^{\infty} R_{l^{\prime}}(r) j_{L}(q r) R_{l}(r) r^{2} d r .
$$

The spin projection integral is discussed in appendix $B$ and has the value

$$
\left[\tilde{\chi}^{[1 / 2]} \mid \chi^{[1 / 2]}\right]=+\frac{\hat{1}}{2}=\sqrt{2}
$$

The other factors on the right-hand side of eq (25) are given by eqs (19) and (22). Useful relationships for reducing the 9-j coefficients when they contain zeros are given in appendix C. The result is

$$
\begin{gathered}
M_{L}(q)=(i)^{\left(l+l-l^{\prime}\right)}(-1)^{j^{\prime}+1 / 2} \frac{\hat{l}^{\prime} \hat{l} \hat{j}^{\prime} \hat{j} \hat{L}}{\sqrt{4 \pi}} \\
\left\{\begin{array}{lll}
l^{\prime} & j^{\prime} & \frac{1}{2} \\
j & l & L
\end{array}\right\}\left(\begin{array}{ccc}
l^{\prime} & L & l \\
0 & 0 & 0
\end{array}\right)\left\langle R_{l^{\prime}}\left|j_{L}(q r)\right| R_{l}\right\rangle .
\end{gathered}
$$

Recoupling applied to other electromagnetic multipole operators is discussed in [8].

\subsection{A Sum Rule for the 6-j Symbol}

Identities and sum rules among the $6-j$ and $9-j$ coefficients can be verified with the recoupling dia- grams. If a recoupling can be accomplished in two different ways (i.e., by two different flowcharts), the two transformations must be equivalent.

Before we begin an example, let us note the useful simplification occurring in a four-element recoupling box when the two outside lines are mock zeros. Figure $7 \mathrm{a}$ is a crossing of the nonzero lines and results in a phase factor for elements that commute:

$$
\left[\begin{array}{lll}
0 & a & a \\
b & 0 & b \\
b & a & c
\end{array}\right]=(-1)^{c-a-b}
$$

Figures $7 \mathrm{~b}$ and $7 \mathrm{c}$ show schemes in which $a b$ and $c d$ are recoupled to $a c$ and $b d$. Figure $7 \mathrm{~b}$ does it with one $9-j$ box while figure $7 c$ does it with three 9-j boxes. Since the transformations are equivalent, independent of wave functions, one has the relation

$$
\left[\begin{array}{lll}
a & b & e \\
c & d & e \\
f & f & 0
\end{array}\right]=\sum_{x}\left[\begin{array}{lll}
b & 0 & b \\
c & d & e \\
x & d & a
\end{array}\right](-)^{x-b-c}\left[\begin{array}{lll}
a & 0 & a \\
c & b & x \\
f & b & d
\end{array}\right]
$$

which reduces to the indentity

$$
\left\{\begin{array}{lll}
a & b & e \\
d & c & f
\end{array}\right\}=\sum_{x}(-1)^{e+f+x}(2 x+1)\left\{\begin{array}{lll}
a & d & x \\
c & b & e
\end{array}\right\}\left\{\begin{array}{lll}
c & b & x \\
d & a & f
\end{array}\right\} .
$$

\subsection{A Product Operator}

This example involves introducing a complete set of intermediate angular states through the

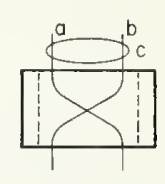

(a)

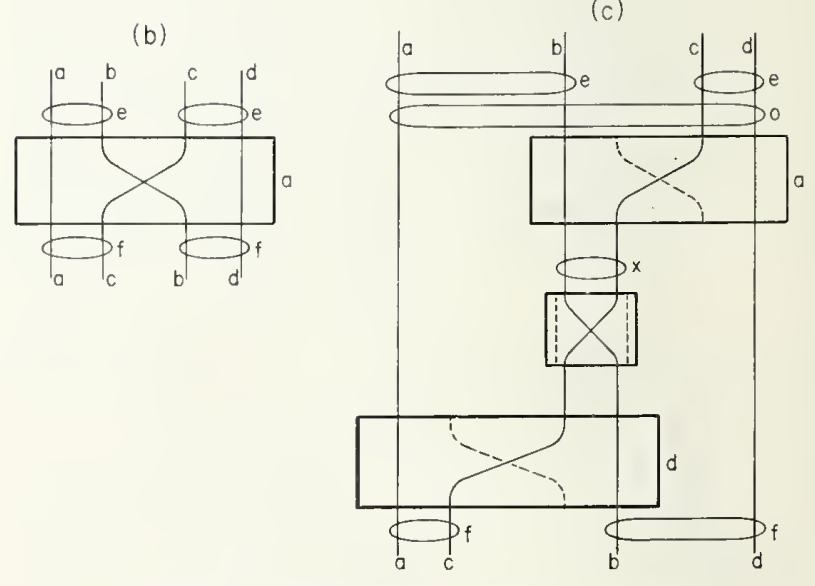

FigURE 7. Flowcharts for proving a 6-j identity. 
closure identity, eq (14). A projection integral that has two coupled operators is evaluated by uncoupling the operators and inserting

$$
\sum_{k \alpha \alpha}(-1)^{2 k} \hat{k}\left[\psi_{\alpha}^{[k]} \times \tilde{\psi}_{\alpha}^{[k]}\right]^{[0]}=\mathbb{1}
$$

between them. The graph for equation (32) is made by combining figure $2 \mathrm{~b}$ and figure $7 \mathrm{a}$. Figure 8 shows how the operators $U^{[L]}$ and $V^{[J]}$ are rccoupled to produce the product of the projection intergrals of each operator. The labeling of the intermediate loops as $j_{1}$ and $j_{2}$ is inferred from the final zero coupling of $\left(j_{2} L k\right)$ and $\left(k J j_{1}\right)$. The flowchart produces the equation

$$
\begin{gathered}
{\left[\tilde{\psi}_{\beta}^{\left[j_{2}\right]}\left|\left[U^{[L]} \times V^{[J]}\right]^{[K]}\right| \psi_{\gamma}^{\left[j_{1}\right]}\right]} \\
=\sum_{k \alpha} \hat{k}(-1)^{2 k}\left[\begin{array}{lll}
k & k & 0 \\
0 & J & J \\
k & j_{1} & J
\end{array}\right]\left[\begin{array}{lll}
L & 0 & L \\
k & j_{1} & J \\
j_{2} & j_{1} & K
\end{array}\right]\left[\begin{array}{lll}
j_{2} & j_{1} & K \\
0 & j_{1} & j_{1} \\
j_{2} & 0 & j_{2}
\end{array}\right] \\
\cdot\left[\tilde{\psi}_{\beta}^{\left[j_{2}\right]}\left|U^{[L]}\right| \psi_{\alpha}^{[K]}\right]\left[\tilde{\psi}_{\alpha}^{[k]}\left|V^{[J]}\right| \psi_{\gamma}^{\left[j_{1}\right]}\right] \\
=(-)^{J+L+j_{1}+j_{2}} \hat{K} \sum_{k \alpha}\left\{\begin{array}{lll}
L & J & K \\
j_{1} & j_{2} & k
\end{array}\right\} \\
\cdot\left[\tilde{\psi}_{\beta}^{\left[j_{2}\right]}\left|U^{[L]}\right| \psi_{\alpha}^{[k]}\right]\left[\tilde{\psi}_{\alpha}^{[k] \mid}\left|V^{[J]}\right| \psi_{\gamma}^{\left[j_{1}\right]}\right] .
\end{gathered}
$$

Examples of such product operators are the orbital angular momentum

$$
\mathbf{L}=\mathbf{r} \times \mathbf{p}=\sqrt{2}\left[r^{[1]} \times p^{[1]}\right]^{[1]}
$$

and the electromagnetic current multipole operator $\left[j_{L}(q r) Y^{[L]} \times p^{[1]}\right]^{[J]}$.

\subsection{An Angular Distribution}

Angular distributions $[9,10]$ play an important role in atomic, nuclear and elementary particle physics, since spins and parities of final states and the nature of intermediate states can be ascertained. The derivation of the appropriate formula is a recoupling problem.

We consider as a specific example a general $(\gamma$, $N$ ) angular distribution as depicted in figure 9. The transition is regarded as a two-step process through the intermediate state, $I_{k}$. The first step is the transition from the ground state, $I_{0}$, to the state, $I_{k}$, by absorption of a photon of multipolarity, $L$. The decay of the state, $I_{k}$, through particle emission involves coupling the emitted particle spin, $s$, and orbital angular momentum, $l$. We do this in the $j$-representation (see fig. 9) as opposed to the channel-spin representation where $\mathbf{I}_{f}+s=\mathbf{S}$ and $\mathbf{S}+\mathbf{l}=\mathbf{I}_{i}$; one representation can be obtained from the other simply by a recoupling.

$$
\left[\widetilde{\psi}_{\beta}^{[i 2]}\left|\left[U^{[L]} \times V^{[J]}\right]^{[K]}\right|_{\psi_{\gamma}^{[j]}}^{[j]}\right.
$$

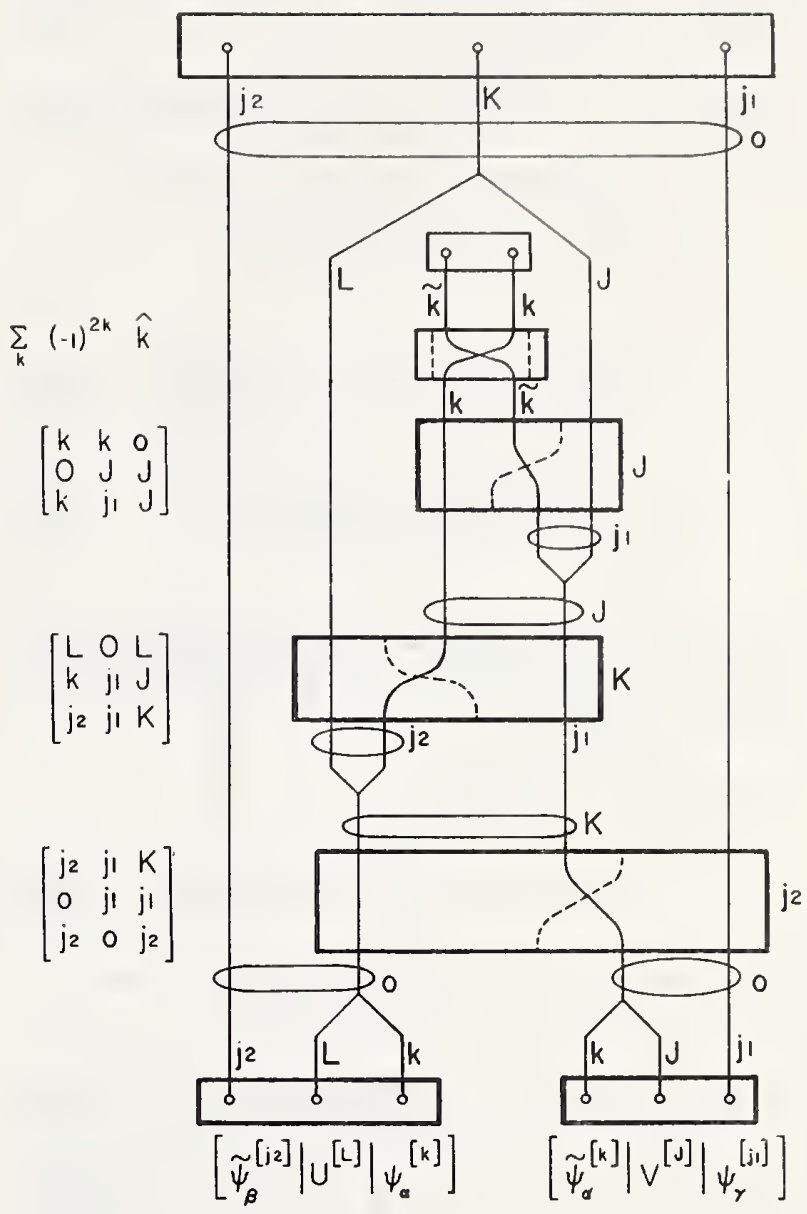

FIGURE 8. Flowchart for separating a product operator.

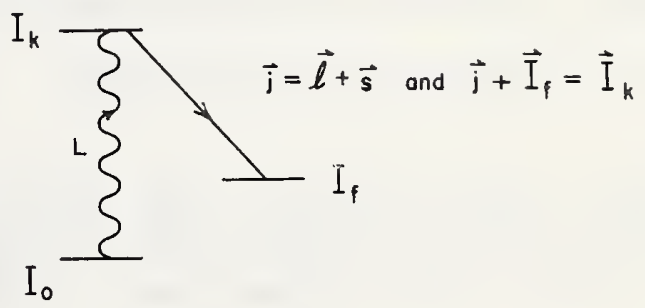

FIGURE 9. Photonuclear angular distribution.

For the initial state, we can write

$$
\psi_{\text {initial }}=\Phi_{m_{0}}^{\left[I_{0}\right]} e^{i k z} \epsilon_{m_{L}}^{[1]},
$$

where $\Phi_{m_{0}}^{[10]}$ represents the ground state and $e^{i k z} \epsilon_{m_{L}}^{[1]}$ the photon incident along the $z$ axis. $\epsilon_{m_{L}}^{[1]}$ is the photon spin function which has two components, $m_{l}= \pm 1$ (transverse-circularly polarized). The 
product $e^{i k z} \epsilon_{m_{L}}^{[1]}$ can be written as the familiar multipole expansion in terms of vector spherical harmonics (appendix C):

$$
\begin{aligned}
e^{i k z} \epsilon_{m_{L}}^{[1]}= & \sum_{L=1}^{\infty}(-1)^{L+1} \hat{L}(2 \pi)^{1 / 2}\left\{m _ { L } j _ { L } ( k r ) \mathscr { Y } \left[L, 1 ; m_{L}\left(\Omega_{r}\right)\right.\right. \\
& +\frac{\sqrt{L}}{\hat{L}} j_{L+1}(k r) \mathscr{Y}_{L+1,1 ; m_{L}}^{[L]}\left(\Omega_{r}\right) \\
& \left.+\frac{\sqrt{L+1}}{\hat{L}} j_{L-1}(k r) \mathscr{Y}_{L-1,1 ; m_{L}}^{[L]}\left(\Omega_{r}\right)\right\} \\
& \equiv \sum_{L=1}^{\infty}(-1)^{L} \hat{L} C_{L}(k r) \Psi_{m_{L}}^{[L]}\left(\Omega_{r}\right) .
\end{aligned}
$$

If eq (37) is substituted into eq (35) and the result coupled to states of total angular momentum, $I_{k}$, eq (35) becomes

$$
\begin{aligned}
\psi_{\text {initial }}=\sum_{L I_{k}}(-1)^{L} \hat{L} C_{L}(k r) \\
\quad\left\langle L m_{L} I_{0} m_{0} \mid I_{k} m_{k}\right\rangle\left[\Psi[L] \times \Phi^{[I 0]}\right]_{m_{k}}^{\left[I_{K}\right]} .
\end{aligned}
$$

Since total angular momentum and parity are conserved, the final state can be written as

$\psi_{\text {final }}=\sum_{L I_{k}}(-1)^{L} \hat{L} A_{L, I_{k}}\left\langle L m_{L} I_{0} m_{0} \mid I_{k} m_{k}\right\rangle \Xi{ }_{m_{k}}^{\left[I_{k}\right]}$,

with $A_{L, I_{k}} \equiv C_{L} M_{I_{k}}$, where $M_{I_{k}}$ is the amplitude for the photoabsorption through the state $I_{k}$. The state $\Xi_{m_{k}}^{\left[I_{k}\right]}$ can now be resolved into its resultant configuration, which in the coordinate representation is

$$
\Xi=(i)^{l}\left[\left[Y^{[l]}\left(\Omega_{N}\right) \times \chi^{[s]}\right]^{[j]} \times \Theta^{\left[I_{f}\right]}\right]_{m_{k}}^{\left[I_{k}\right]},
$$

where the phase $(i)^{l}$ comes from using contrastandard spherical harmonics. The angular distribution of the outgoing particle with respect to the incident photon direction (the $z$ axis) is

$\int \psi_{\text {final }}^{\dagger} \psi_{\text {final }}$ averaged over initial spins. The $\int$

means do all spin-coordinate integrations, except those over the nucleon's angular coordinates. Explicitly, we have

$W(\theta)=\frac{1}{2 \hat{I_{0}^{2}}} \sum_{L L^{\prime} I_{k} I_{k}^{\prime}} \sum_{m_{0} m_{L}= \pm 1}(-1)^{L+L^{\prime}+\left(l-l^{\prime}\right) / 2} \int$

$\cdot \hat{L} \hat{L}^{\prime} A_{L^{\prime}, I_{k}^{\prime}}^{\dagger} A_{L, I_{k}}\left\langle L^{\prime} m_{L} I_{0} m_{0} \mid I_{k}^{\prime} m_{k}\right\rangle\left\langle L m_{L} I_{0} m_{0} \mid I_{k} m_{k}\right\rangle$
$\cdot\left[\left[Y^{\left[l^{\prime}\right]} \times \tilde{\chi}^{[s]}\right]^{\left[j^{\prime}\right]}\right.$

$$
\left.\times \tilde{\Theta}^{\left[I_{f}\right.}\right]_{m_{k}}^{\left.\left[l_{k}\right]\right]^{C}}\left[\left[Y^{[l]} \times \chi^{[8]}\right]^{[j]} \times \Theta^{\left[I_{f}\right]}\right]_{m_{k}}^{\left[I_{k}\right]} .
$$

The primed variables allow for interference. Using the conjugation property, eq $(A-2)$, brings in a phase $(-1)^{I} k^{+}+m_{k}$, and then we couple the last two factors to angular momentum $\lambda$ :

$$
\begin{aligned}
& W(\theta)=\frac{1}{2 \hat{I}_{0}^{2}} \sum_{L L^{\prime} l_{k} I_{k}^{\prime} \lambda} \sum_{m_{0} m_{L}= \pm 1}(-1)^{L+L^{\prime}+\left(l-l^{\prime}\right) / 2+I_{k}^{\prime}+m_{k}} \int \\
& \cdot \hat{L} \hat{L}^{\prime} A_{L^{\prime} I_{k}^{\prime}}^{\dagger} A_{l, I_{k}}\left\langle L^{\prime} m_{L} I_{0} m_{0} \mid I_{k}^{\prime} m_{k}\right\rangle\left\langle L m_{L} I_{0} m_{0} \mid I_{k} m_{k}\right\rangle \\
& \cdot\left\langle I_{k}^{\prime}-m_{k} I_{k} m_{k} \mid \lambda 0\right\rangle\left[[\ldots]^{\left[I_{k}^{\prime}\right]} \times[\ldots]^{\left[I_{k}\right]}\right]_{0}^{[\lambda]} .
\end{aligned}
$$

The problem now amounts to simplifying eq (42).

The simplification of eq (42) is done in two steps: (1) Removal of the spin functions and (2) Recombination of the three vector-coupling coefficients.

$$
\left.\left[\left[\left[Y^{\left[\ell^{\prime}\right]} \times \widetilde{X}^{[s]}\right]^{\left[j^{\prime}\right]} \times \widetilde{\Theta}^{\left[I_{k}\right]}\right]_{m_{k}}^{\left[I_{k}^{\prime}\right]} \times\left[\left[Y^{[l]} \times X^{[s]}\right]^{[j]} \times \Theta^{\left[I_{f}\right]}\right]\right]_{m_{k}}^{\left[I_{k}\right]}\right]_{0}^{[\lambda]}
$$

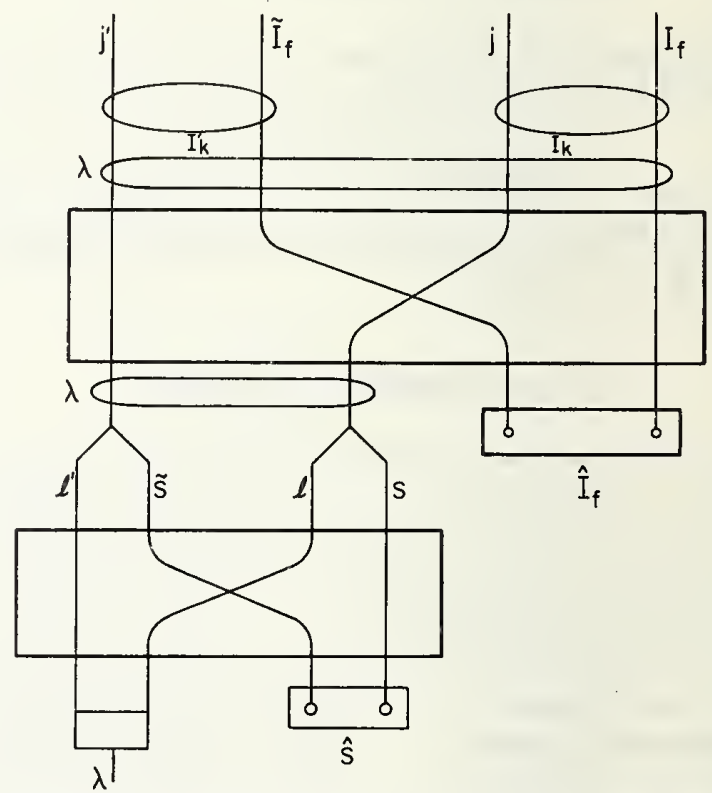

FIgURE 10. Recoupling the angular distribution formula.

Removal of the spin functions can be achieved with the flowchart in figure 10. Using the rules established above, we get

$$
\int\left[[\ldots]\left[I_{k}^{\prime}\right] \times[\ldots]^{\left[I_{k}\right]}\right]_{0}^{[\lambda]}
$$




$$
=\left[\begin{array}{ccc}
j^{\prime} & I_{f} & I_{k}^{\prime} \\
j & I_{f} & I_{k} \\
\lambda & 0 & \lambda
\end{array}\right]\left[\begin{array}{lll}
l^{\prime} & s & j^{\prime} \\
l & s & j \\
\lambda & 0 & \lambda
\end{array}\right] \hat{I}_{f} \hat{s} Q_{l^{\prime} l}^{[\lambda]} Y_{0}^{[\lambda]}\left(\Omega_{N}\right),
$$

which reduces to

$$
\begin{gathered}
=(-1)^{j^{\prime}+j+s+I_{f}+I_{k}+\left(l-l^{\prime}\right) / 2} \hat{j} \hat{j}^{\prime} \hat{I}_{k} \hat{I}_{k}^{\prime} \hat{\lambda} \hat{l} \hat{l}^{\prime} . \\
\left\{\begin{array}{lll}
j & j^{\prime} & \lambda \\
I_{k}^{\prime} & I_{k} & I_{f}
\end{array}\right\}\left\{\begin{array}{lll}
l & l^{\prime} & \lambda \\
j^{\prime} & j & s
\end{array}\right\}\left(\begin{array}{lll}
l & l^{\prime} & \lambda \\
0 & 0 & 0
\end{array}\right) \frac{1}{4 \pi} P_{\lambda}(\cos \theta),
\end{gathered}
$$

where we have used $Y_{0}^{[\lambda]}\left(\Omega_{N}\right)=(-1)^{-\lambda / 2} \hat{\lambda} P_{\lambda}(\cos \theta) /$ $(4 \pi)^{1 / 2}$. If the spin of the ejected particle is $\frac{1}{2}$, eq (44) can be simplified to

$$
\begin{gathered}
=(-1)^{j^{\prime}+j-1 / 2+I}{ }_{f}^{+I_{k}+1 / 2(l-l)} \hat{j}^{j^{\prime}} \hat{I}_{k} \hat{I}_{k}^{\prime} \hat{\lambda} \\
\left\{\begin{array}{lll}
j & j^{\prime} & \lambda \\
I_{k}^{\prime} & I_{k} & I_{f}
\end{array}\right\}\left(\begin{array}{ccc}
j & j^{\prime} & \lambda \\
\frac{1}{2} & -\frac{1}{2} & 0
\end{array}\right) \frac{1}{4 \pi} P_{\lambda}(\cos \theta),
\end{gathered}
$$

with the identity

$$
\left\{\begin{array}{lll}
l & l^{\prime} & \lambda \\
j^{\prime} & j & \frac{1}{2}
\end{array}\right\}\left(\begin{array}{lll}
l & l^{\prime} & \lambda \\
0 & 0 & 0
\end{array}\right)=-\frac{1}{\hat{l} \hat{l}^{\prime}}\left(\begin{array}{rrr}
j & j^{\prime} & \lambda \\
\frac{1}{2} & -\frac{1}{2} & 0
\end{array}\right) .
$$

Recombination of the vector-coupling coefficients can be achieved in several ways. We could write them as $3-j$ coefficients and manipulate the order of their arguments until use can be made of the identity which relates a sum over the product of three 3-j's to the product of a $6-j$ and a $3-j$ (See Edmond's eq (6.2.8, p. 95) [7]). However, one can also recombine them with the aid of a diagram. This combination of vector-coupling coefficients is like that which occurs in the coupling (with arbitrary functions)

$$
\begin{gathered}
{\left[\left[\Phi^{\left[L^{\prime}\right]} \times \psi^{[I 0]}\right]^{\left[I_{k}^{\prime}\right]} \times\left[\chi^{[L]} \times \Sigma^{[I 0]}\right]^{\left[I_{k}\right]}\right]_{0}^{[\lambda]}} \\
=\sum_{\substack{m_{k} \\
m_{L}^{\prime} m_{0}^{\prime} \\
m_{L} m_{0}}}\left\langle L^{\prime} m_{L}^{\prime} I_{0} m_{0}^{\prime} \mid I_{k}^{\prime}-m_{k}\right\rangle\left\langle L m_{l^{\prime}} I_{0} m_{0} \mid I_{k} m_{k}\right\rangle \\
\quad\left\langle I_{k}^{\prime}-m_{k} I_{k} m_{k} \mid \lambda 0\right\rangle \Phi_{m_{\ell}}^{\left.L \prime^{\prime}\right]} \psi_{m_{0}^{\prime}}^{[I 0]} \chi_{m_{l}}^{[L]} \sum_{m_{0}}^{(I 0)},
\end{gathered}
$$

which can be recoupled to

$$
=\sum_{J J^{\prime}}\left[\begin{array}{ccc}
L^{\prime} & I_{0} & I_{k}^{\prime} \\
L & I_{0} & I_{k} \\
J^{\prime} & J & \lambda
\end{array}\right]\left[\left[\Phi^{\left[L^{\prime}\right]} \times \chi^{[L]}\right]^{\left[J^{\prime}\right]} \times\left[\psi^{[/ 0]} \times \Sigma^{[I 0]}\right]^{[J]}\right]_{0}^{[\lambda]}
$$

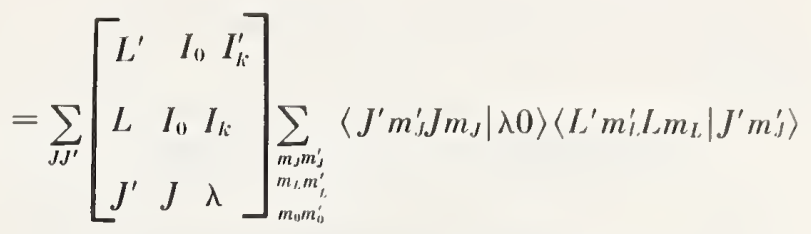$$
\left\langle I_{0} m_{0}^{\prime} I_{0} m_{0} \mid J m_{J}\right\rangle \Phi_{m_{l}^{\prime}}^{\left[I^{\prime} \mid\right.} \chi_{m_{l}}^{\left[I_{l} \mid\right.} \psi_{m_{l_{0}}}^{\left[I_{0}\right]} \sum_{m_{0}}^{\left[I_{0}\right]}
$$

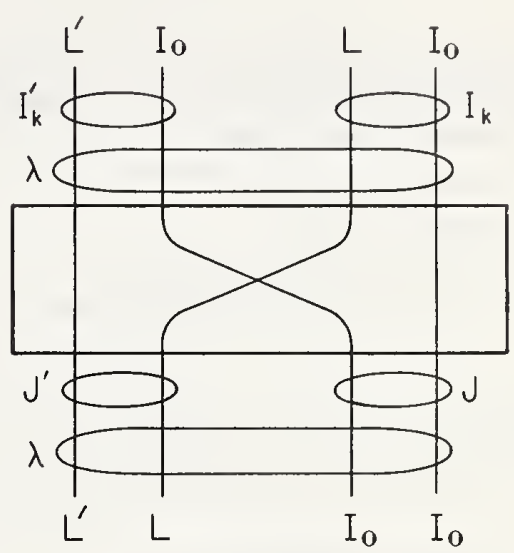

FIGURE 11. Recoupling graph to obtain a vector-coupling identity.

with the diagram in figure 11. Since the functions $\Phi, \chi, \psi$, and $\Sigma$ are arbitrary and the sums over $m_{L}$, $m_{L}^{\prime}, m_{0}$, and $m_{0}^{\prime}$ appear on both sides, we have the identity

$\sum_{m_{k}}\left\langle L^{\prime} m_{L}^{\prime} I_{0} m_{0}^{\prime} \mid I_{k}^{\prime}-m_{k}\right\rangle\left\langle L m_{L} I_{0} m_{0} \mid I_{k} m_{k}\right\rangle\left\langle I_{k}^{\prime}\right.$

$$
-m_{k} I_{k} m_{k}|\lambda 0\rangle
$$

$$
\begin{gathered}
=\sum_{\substack{J J^{\prime} \\
m m_{J}^{\prime}}}\left[\begin{array}{ccc}
L^{\prime} & I_{0} & I_{k}^{\prime} \\
L & I_{0} & I_{k} \\
J^{\prime} & J & \lambda
\end{array}\right]\left\langle J^{\prime} m_{J}^{\prime} J m_{J} \mid \lambda 0\right\rangle\left\langle L^{\prime} m_{L}^{\prime} L m_{L} \mid J^{\prime} m_{J}^{\prime}\right\rangle \\
\cdot\left\langle I_{0} m_{0}^{\prime} I_{0} m_{0} \mid J m_{J}\right\rangle
\end{gathered}
$$

Equation (50) is put into the form we want by four steps: (1) Let $m_{0}^{\prime}=-m_{0}$ which implies $m_{L}^{\prime}=-m_{L}$ and $m_{J}=m_{J}{ }_{J}=0$. (2) Multiply both sides by $\left\langle I_{0}-\right.$ $m_{0} I_{0} m_{0}|00\rangle=(-1)^{I_{0}+m_{0} / \hat{I}_{0}}$ and sum over $m_{0}$. (3) On the right-hand side use $\sum_{m_{0}}\left\langle I_{0}-m_{0} I_{0} m_{0}\right| \begin{array}{lll}0 & 0\rangle\left\langle I_{0}-\right.\end{array}$ $m_{0} I_{0} m_{0}|J 0\rangle=\delta_{J 0}$, which, since $J=0$, implies $J^{\prime}=\lambda$. (4) Sum over $m_{L}= \pm 1$ and simplify the right-hand side. We then have 
$\sum_{\substack{m_{k} m_{0} \\ m_{L}= \pm 1}}(-1)^{I 0+m_{0}}\left(\hat{I}_{0}\right)^{-1}\left\langle L^{\prime}-m_{L} I_{0}-m_{0} \mid I_{k}^{\prime}-m_{k}\right\rangle$

$$
\begin{gathered}
\left\langle L m_{L} I_{0} m_{0} \mid I_{k} m_{k}\right\rangle \cdot\left\langle I_{k}^{\prime}-m_{k} I_{k} m_{k} \mid \lambda 0\right\rangle \\
=(-1)^{L^{\prime}+I_{0}+I_{k}} \frac{2 \hat{I}_{k} \hat{I}_{k}^{\prime} \hat{\lambda}}{\hat{I}_{0}}\left\{\begin{array}{lll}
L & L^{\prime} & \lambda \\
I_{k}^{\prime} & I_{k} & I_{0}
\end{array}\right\}\left(\begin{array}{lll}
L & L^{\prime} & \lambda \\
-1 & 1 & 0
\end{array}\right) .
\end{gathered}
$$

Therefore, the combination of vector-coupling coefficients appearing in eq (42) can be rewritten by use of eq (51) and the facts that

$$
\begin{aligned}
& \left\langle L^{\prime}-m_{L} I_{0}-m_{0} \mid I_{k}^{\prime}-m_{k}\right\rangle \\
& =(-1)^{L^{\prime}+I_{0}-I_{k}^{\prime}}\left\langle L^{\prime} m_{L} I_{0} m_{0} \mid I_{k}^{\prime} m_{k}\right\rangle \\
& \text { and }(-1) m_{L}=-1 .
\end{aligned}
$$

The result is

$$
\sum_{\substack{m_{0} \\ m_{L}= \pm 1}}(-1)^{m_{k}}\left\langle L^{\prime} m_{L} I_{0} m_{0} \mid I_{k}^{\prime} m_{k}\right\rangle
$$

$$
\begin{gathered}
\left\langle L m_{L} I_{0} m_{0} \mid I_{k} m_{k}\right\rangle\left\langle I_{k}^{\prime}-m_{k} I_{k} m_{k} \mid \lambda 0\right\rangle \\
=(-1)^{I 0+I_{k}-I k^{\prime}+1} 2 \hat{I}_{k} \hat{I}_{k}^{\prime} \hat{\lambda}\left\{\begin{array}{lll}
L & L^{\prime} & \lambda \\
I_{k}^{\prime} & I_{k} & I_{0}
\end{array}\right\}\left(\begin{array}{ccc}
L & L^{\prime} & \lambda \\
-1 & 1 & 0
\end{array}\right) .
\end{gathered}
$$

Substituting eqs (45) and (52) into eq (42), as an example, we get the $\gamma$-nucleon angular distribution formula

$$
\begin{aligned}
& W(\theta)=\sum_{\substack{L, L^{\prime}}} \sum_{\substack{I_{k} \\
l_{k} \\
\lambda^{\prime}}}^{\dagger} A_{L^{\prime}, I_{h}^{\prime}}^{+} A_{L, I_{k}} \\
& \cdot\left[(-1)^{I_{k}-I_{0}+1} \hat{\lambda} \hat{L} \hat{L}^{\prime} \hat{I}_{k} \hat{I}_{k}^{\prime}\left\{\begin{array}{lll}
L & L^{\prime} & \lambda \\
I_{k}^{\prime} & I_{k} & I_{0}
\end{array}\right\}\left(\begin{array}{lll}
I & L^{\prime} & \lambda \\
-1 & 1 & 0
\end{array}\right)\right] \\
& \cdot\left[(-1)_{k}^{I_{k}-I_{f}-j-j^{\prime}+1 / 2} \hat{I}_{k} \hat{I}_{k}^{\prime} \hat{j} \hat{j}^{\prime} \hat{\lambda}\left\{\begin{array}{lll}
j & j^{\prime} & \lambda \\
I_{k}^{\prime} & I_{k} & I_{f}
\end{array}\right\}\right. \\
& \left.\cdot\left(\begin{array}{lr}
j & j^{\prime} \lambda \\
\frac{1}{2}-\frac{1}{2} & 0
\end{array}\right)\right] P_{\lambda}(\cos \theta),
\end{aligned}
$$


Obtain the result

$\left[\tilde{\psi}^{\left[j^{\prime}\right]}\left|j_{L}\left[Y^{[L]} \times \sigma^{[1]}\right]^{[J]}\right| \psi^{[j]}\right]$

$$
\begin{aligned}
& =(-1)^{j-J-j^{\prime}+\left(l^{\prime}+L+1+l\right) / 2}\left[\frac{6}{4 \pi}\right]^{1 / 2} \hat{J} \hat{l}_{j^{\prime}} \hat{j}^{\prime} \hat{l}^{\prime} \hat{l} \\
& \left\{\begin{array}{lll}
l^{\prime} & l & L \\
\frac{1}{2} & \frac{1}{2} & 1 \\
j^{\prime} & j & J
\end{array}\right\}\left(\begin{array}{lll}
l^{\prime} & L & l \\
0 & 0 & 0
\end{array}\right)\left\langle R_{l^{\prime}}\left|j_{l}\right| R_{l}\right\rangle .
\end{aligned}
$$

\subsection{The Normalization Condition for the $9-j$ and $6-j$ Coefficients}

Prove the equation

$$
\begin{gathered}
\sum_{J_{13}, J_{24}}\left(2 J_{13}+1\right)\left(2 J_{24}+1\right) \quad\left\{\begin{array}{lll}
j_{1} & j_{2} & J_{12} \\
j_{3} & j_{4} & J_{34} \\
J_{13} & J_{24} & J
\end{array}\right\}^{2} \\
=\frac{1}{\left(2 J_{12}+1\right)\left(2 J_{34}+1\right)}
\end{gathered}
$$

by recoupling the two pairs $\left(j_{1}, j_{2}\right)$ and $\left(j_{3}, j_{4}\right)$ from $J_{12}$ and $J_{34}$ to $J_{13}$ and $J_{24}$, respectively, with one box, and then recouple with a second box back to the original wave function with intermediate momenta $J_{12}^{\prime}$ and $J_{34}^{\prime}$. The total angular momentum is taken as $J$.

When $J_{12}^{\prime}=J_{12}$ and $J_{34}^{\prime}=J_{34}$, the transformation must equal unity since it reproduces the original wave function.

Show by recoupling that the $6-j$ coefficient normalization is

$$
\sum_{J_{1}}\left(2 J_{1}+1\right)\left\{\begin{array}{lll}
j_{1} & j_{2} & J_{1} \\
j_{4} & j_{3} & J_{2}
\end{array}\right\}^{2}=\frac{1}{2 J_{2}+1}
$$

where $J_{1}$ and $J_{2}$ are the pair momenta.

\subsection{A $\left(\gamma, \gamma^{\prime}\right)(A-1)+N$ Angular Distribution}

A problem which is of current experimental interest is that of photon absorption with subsequent photon + nucleon emission. The process can be visualized as taking place in three steps as depicted in figure 12. Experimentally, the emitted nucleon goes undetected and the angular distribution of the emitted photon is measured. Show by methods similar to those in Example D that, after integrating over the nucleon coordinates and removing its spin functions, the emitted-photon angular distribution is given by

$$
\begin{aligned}
& \mathbb{W}(\theta)=\sum_{\substack{l, I_{k} \\
L^{\prime} I^{\prime}{ }_{k} \\
\lambda}} A_{l^{\prime}, I_{h}^{\prime}} A_{l, I_{k}} \\
& \cdot\left[(-1)^{I_{k}-I_{0+1}} \hat{\lambda} \hat{L} \hat{L}^{\prime} \hat{I} \hat{I}_{k}^{\prime}\left\{\begin{array}{lll}
L & L^{\prime} & \lambda \\
I_{k}^{\prime} & I_{k} & I_{0}
\end{array}\right\}\left(\begin{array}{lll}
L & L^{\prime} & \lambda \\
-1 & 1 & 0
\end{array}\right)\right] \\
& {\left[(-1) I_{k}^{\prime}+j+I_{1} \hat{I}_{k} \hat{I}_{k,}^{\prime}\left\{\begin{array}{lll}
I_{k} & I_{k}^{\prime} & \lambda \\
I_{l}^{\prime} & I_{l} & j
\end{array}\right\}\right]} \\
& {\left[(-1)^{l_{1-I_{f}+1}} \hat{\lambda} \hat{I}_{l}^{\prime} \hat{I}_{\mathcal{L}^{\prime}} \hat{\mathcal{L}}^{\prime} \hat{\mathscr{L}}\left\{\begin{array}{lll}
\mathscr{L} & \mathscr{L}^{\prime} & \lambda \\
I_{l}^{\prime} & I_{l} & I_{f}
\end{array}\right\}\right.} \\
& \left.\left(\begin{array}{ccc}
\mathscr{L} & \mathscr{L}^{\prime} & \lambda \\
1 & -1 & 0
\end{array}\right)\right] P_{\lambda}(\cos \theta),
\end{aligned}
$$

where $\theta$ is the angle between the incident and emitted photons. (Hint: the following identity is useful in handling the emitted-photon step

$$
\hat{\mathscr{L}} \hat{\mathscr{L}}\left\{\begin{array}{lll}
\mathscr{L}^{\prime} & \mathscr{L} & \lambda \\
\mathscr{L} & \mathscr{L}^{\prime} & 1
\end{array}\right\}\left(\begin{array}{lll}
\mathscr{L} & \mathscr{L}^{\prime} & \lambda \\
0 & 0 & 0
\end{array}\right)=\left(\begin{array}{ccc}
\mathscr{L}^{\prime} & \mathscr{L} & \lambda \\
1 & -1 & 0
\end{array}\right) .
$$

\subsection{A Two-Body Matrix Element}

Draw the recoupling flowchart that separates a two-body operator sandwiched between two-body wave functions into a product of one-body matrix elements, i.e., show

$$
\begin{aligned}
{\left[\left[\tilde{\varphi}^{\left[j_{1}\right]}(1) \times \tilde{\varphi}^{\left[j_{2}\right]}(2)\right]^{[, f]} \mid\left[T^{[/ 1]}(1)\right.\right.} & \\
& \left.\left.\times T^{\left[l_{2}\right]}(2)\right]^{[/]} \mid\left[\varphi^{[k 1]}(1) \times \varphi^{[k 2]}(2)\right]^{\left[k^{\prime}\right]}\right] \\
\quad & \hat{J} \hat{L} \hat{K}\left\{\begin{array}{lll}
j_{1} & j_{2} & J \\
l_{1} & l_{2} & L \\
k_{1} & k_{2} & K
\end{array}\right\}\left[\tilde{\varphi}^{[j 1]}(1)\left|T^{[/ 1]}(1)\right| \varphi^{\left.\mid k_{1}\right]}(1)\right]
\end{aligned}
$$

$$
\text { · }\left[\tilde{\varphi}^{[j 2]}(2)\left|T^{[/ 2]}(2)\right| \varphi^{\left[k_{2}\right]}(2)\right] \text {. }
$$

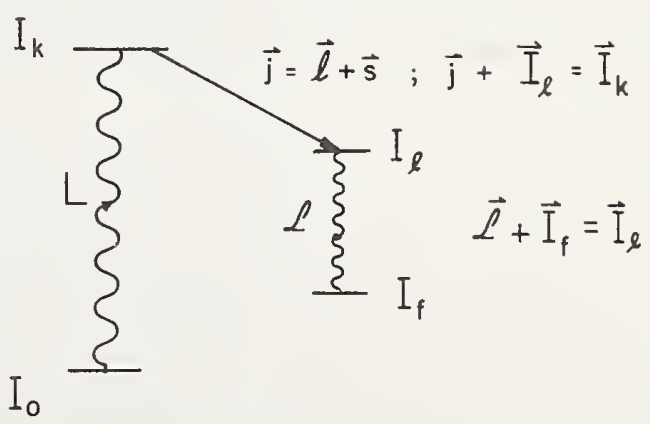

Figure 12. The $\mathrm{A}\left(\gamma, \gamma^{\prime}\right)(\mathrm{A}-1)+\mathrm{N}$ process. 
All recoupling identities using conventional elements (e.g., $\left.\psi_{j m}\right)$ are also true for the contrastandard elements $\left(\psi_{m}^{[j]}\right)$ because the difference in phase conventions appears on both sides of the identities and therefore cancels. To check reduced matrix element identities with recoupling diagrams, one must remember to convert reduced matrix elements to projection integrals using eq (16).

\subsection{A 6-j Sum Rule}

Derive the sum rule

$$
\sum_{x}(-1)^{2 x}(2 x+1)\left\{\begin{array}{lll}
a & b & x \\
a & b & c
\end{array}\right\}=1
$$

by recoupling from

$$
\left[[a \times a]^{[0]} \times[b \times b]^{[0]}\right]^{[0]}
$$

to

$$
\left[[a \times b]^{[c]} \times[a \times b]^{[c]}\right]^{[0]}
$$

by two different flowcharts.

\section{Appendix A: The Projection Integral}

The projection integral introduced by Danos is the quantity to which the tools of Racah algebra are directly applicable. It is related to a general matrix element in a manner similar to the reduced matrix element, but with essential differences.

Consider the matrix element

$$
\mathscr{M}=\int \psi_{M_{1}}^{\left[J_{1}\right]^{\dagger}} T_{m}^{[K]} \Phi_{M_{2}}^{\left[J_{2}\right]}
$$

where, in general, $\psi$ and $T$ represent many-body wave functions and operators, respectively. The angular momentum notation is that of Fano and Racah [12] with $\dagger$. meaning Hermitian conjugate, i.e., $\psi t=\tilde{\psi}^{c}$. The transpose operation, $\sim$. which affects only the half-integral angular momentum, will be discussed in appendix B. Conjugation [13] is defined as

$$
\psi_{M}^{[J]}{ }^{C}=(-1)^{J+M} \psi_{-M}^{[J]},
$$

for both integral and half-integral values of $J$. Equation $(\mathrm{A}-1)$ can then be written as

$$
\mathscr{M}=(-1)^{J 1+M_{1}} \int \tilde{\psi}_{-M_{1}}^{\left[J_{1}\right]} T_{m}^{[K]} \Phi_{M_{2}}^{\left[J_{2}\right]} .
$$

The product of three contrastandard tensor components under the integral can be expanded in terms of angular momentum eigenfunctions

$$
\begin{aligned}
& \underset{-M_{1}}{\left[J_{1}\right]} T_{m}^{[K]} \Phi_{M_{2}}^{\left[J_{2}\right]}=\sum_{I, L} \\
& \left\langle J_{1}-M_{1} L m+H_{2} \mid I m_{1}\right\rangle\left\langle K m J_{2} H_{2} \mid L m+H_{2}\right\rangle \\
& \cdot\left[\tilde{\psi}^{[J 1]} \times\left[T^{[K]} \times \Phi^{\left[J_{2}\right]}\right]^{[L]}\right]_{m_{I}}^{[I]},
\end{aligned}
$$

which reduces to only the $I=0$ term when the angular integration and spin algebra have been completed. The coefficient of this term can be written in terms of the Wigner 3-j coefficient as [14]

$$
\mathscr{M}=(-1)^{J 1+M_{1}}(-1)^{2 K}(-1)^{J 1+K+J_{2}}
$$

$$
\left(\begin{array}{rrr}
J_{1} & K & J_{2} \\
-M_{1} & m & M_{2}
\end{array}\right)
$$

$$
\cdot \int\left[\tilde{\psi}^{\left[J_{1}\right]} \times T^{\left[k^{\prime}\right]} \times \Phi^{\left[J_{2}\right]}\right]^{[0]} .
$$

The integral of the invariant triple product is a projection integral denoted by

$$
\left[\tilde{\psi}^{\left[J_{1}\right]}\left|T^{[K]}\right| \Phi^{\left[J_{2}\right]}\right]=\int\left[\tilde{\psi}^{\left[J_{1}\right]} \times T^{[K]} \times \Phi^{\left[J_{2}\right]}\right]^{[0]} .
$$

The same matrix element expressed in terms of the reduced matrix element as defined by Edmonds, Racah and Wigner is (see Edmonds, p. 88) [7]

$$
\begin{array}{r}
\mathscr{U}=(-1)^{J_{1}-M_{1}}\left(\begin{array}{ccc}
J_{1} & K & J_{2} \\
-M_{1} & m & M_{2}
\end{array}\right) \\
\left\langle\psi^{\left[J_{1}\right]}\left\|T^{[K]}\right\| \Phi^{\left[J_{2}\right]}\right\rangle,
\end{array}
$$

thus

$$
\begin{aligned}
& {\left[\tilde{\psi}^{\left[J_{1}\right]}\left|T^{[K]}\right| \Phi^{\left[J_{2}\right]}\right]} \\
& \quad=(-1)^{J_{1+K-J 2}}\left\langle\psi^{\left[J_{1}\right]}\left\|T^{[K]}\right\| \Phi^{\left[J_{2}\right]}\right\rangle .
\end{aligned}
$$

The main differences between these two quantities are the fact that the projection integral can be used directly in recoupling algebra without phases to worry about, and, in the projection integral, operators and state functions are treated on equal footing.

\section{Appendix B: Half-Integral Angular Momentum}

The case of half-integral angular momentum deserves special treatment, since it illustrates the meaning of conjugation, eq $\left(A^{-}-2\right)$, and permits us 
to derive a relationship between ordinary spin- $-\frac{1}{2}$ eigenstates and the contrastandard ones. We begin by considering the conjugation property.

The conjugation property defines the costandard sets in terms of the contrastandard sets (see FanoRacah, p. 23) [12]

$$
\psi_{M}^{(J)}=\psi_{M}^{|J|^{C}}=(-1)^{J+M} \psi_{-, M}^{[J\}},
$$

where super ( ) denote costandard. For our purposes, it is the rule for replacing all costandard elements involved in recoupling by contrastandard forms. We know that the integral angular momentum eigenfunctions $Y_{m ! l}^{[l]}(\Omega)$, satisfy eq $(B-1)$, if the conjugation operation means complex conjugation. However, this does not apply to the halfintegral angular momentum case.

Consider conjugation to be an operator. $U$. such that

$$
\psi_{., J}^{[J]^{C}}=U \psi_{., y}^{[J]}=(-1)^{J+M} \psi_{-, . M}^{[J]}
$$

and

$$
U \psi_{M}^{[J]^{C}} \equiv U^{2} \psi_{M}^{[J]}=(-1)^{2 J} \psi_{M}^{[J]} .
$$

Only for $J$ an integer can $U$ be interpreted as complex conjugation. When $J$ is a half-integer, $U$ must have another meaning compatible with eq $(\mathrm{B}-3)$.

We uncover the meaning of $U$ for half-integral angular momentum by specializing to the $\operatorname{spin}-\frac{1}{2}$ case. We choose the representation in which the third Pauli matrix is diagonal and thus

$$
\chi_{1 / 2}^{[1 / 2]}=\left(\begin{array}{l}
1 \\
0
\end{array}\right) ; \quad \chi_{-1 / 2}^{[1 / 2]}=\left(\begin{array}{l}
0 \\
1
\end{array}\right) .
$$

Then by the conjugation property eq $(B-1)$, we get

$$
\chi_{1 / 2}^{[1 / 2]}{ }^{C}=-\left(\begin{array}{l}
0 \\
1
\end{array}\right) ; \quad \chi_{-1 / 2}^{[1 / 2]^{C}}=\left(\begin{array}{l}
1 \\
0
\end{array}\right) .
$$

If we impose orthonormality, $\tilde{\chi}_{\mu}^{C} \chi_{\mu^{\prime}}=\delta_{\mu \mu^{\prime}}$, by use of the ordinary definition of matrix transpose, a contradiction arises. One way out of this impasse is to introduce more freedom into the problem, for example, let the transposed quantities corresponding to eq $(B-4)$ be

$$
\tilde{\chi}_{1 / 2}^{[1 / 2]}=\left(\begin{array}{ll}
\alpha \beta
\end{array}\right) ; \quad \tilde{\chi}_{-1 / 2}^{[1 / 2]}=\left(\begin{array}{ll}
\gamma & \delta
\end{array}\right),
$$

with $\alpha, \beta, \gamma, \delta$ real constants. If we assume the transposed quantities conjugate by eq $(\mathrm{B}-1)$, orthonormality now gives

$$
\begin{aligned}
& \alpha=0 \\
& \beta=1 \\
& \gamma=-1 \\
& \delta=0 .
\end{aligned}
$$

Explicitly,

$$
\begin{aligned}
& \tilde{\chi}_{1 / 2}^{[1 / 2]}=\left(\begin{array}{ll}
0 & 1
\end{array}\right) ; \quad \tilde{\chi}_{-1 / 2}^{11 / 2]}=\left(\begin{array}{ll}
-1 & 0
\end{array}\right) \quad(B-8) \\
& \tilde{\chi}_{1 / 2}^{11 / 2]^{C}}=\left(\begin{array}{ll}
1 & 0
\end{array}\right) ; \quad \tilde{\chi}_{-1 / 2}^{[1 / 2)^{C}}=\left(\begin{array}{ll}
0 & 1
\end{array}\right) . \quad(B-9)
\end{aligned}
$$

The meaning of the conjugation operator for this representation is clear:

$$
U=i \sigma_{2}=\left(\begin{array}{rr}
0 & 1 \\
-1 & 0
\end{array}\right)
$$

where $\sigma_{2}$ is the second Pauli matrix. In general, $U$ is the Fano-Racah $U$ matrix, which corresponds to a rotation about the $y$-axis by $\pi$ radians [15]. Specifically, $U$ is the operation $d_{M^{\prime}, M}^{[J]}(\pi)=(-1)^{J+M} \delta_{M^{\prime},-M}$

The main point of the preceding paragraph is that no phase is needed when relating the contrastandard spinors to the ordinary spin- $-\frac{1}{2}$ spinors. In actual practice an explicit representation is not needed. We want simply

$$
\left\langle\chi_{\mu^{\prime}}^{[j]}\left|1_{0}^{[0]}\right| \chi_{\mu}^{[j]}\right\rangle=\delta_{\mu^{\prime} \mu}
$$

for half-integral $j$. We define

and

$$
\left|\chi_{\mu}^{[j]}\right\rangle=\chi_{\mu}^{[j]}
$$

$$
\left\langle\chi_{\mu}^{[j]}\right|=\tilde{\chi}_{\mu}^{[j]^{C}}=(-1)^{j+\mu} \tilde{\chi}_{-\mu}^{[j]} .
$$

From appendix A, eq $\left(\mathrm{B}^{-13}\right)$ becomes

$$
\left.\left\langle\chi_{\mu^{\prime}}^{[j]}\right|\right]_{0}^{[0]}\left|\chi_{\mu}^{[j]}\right\rangle=\frac{1}{\hat{j}}\left[\tilde{\chi}^{[j]} \mid \chi^{[j]}\right] \delta_{\mu^{\prime} \mu}
$$

and thus

$$
\left[\tilde{\chi}^{[j]} \mid \chi^{[j]}\right]=\hat{j},
$$

where $\hat{j}=(2 j+1)^{1 / 2}$. The projection integral, eq $(B-15)$, is the important quantity in recoupling, not the explicit form of $\chi^{[j]}$.

\section{Appendix C: Useful Relations}

We collect a number of standard formulas needed to work the exercises and to make this article selfcontained.

\section{9-j Coefficients}

The $9-j$ coefficients are invariant under an even number of permutations of rows or columns and under transposition about one of the diagonals. An odd number of permutations of rows or columns introduces a phase of minus one to the sum of all nine indices. A 9.j coefficient with a zero as one 
element can be reduced to a $6-j$ coefficient:

$$
\left\{\begin{array}{lll}
j_{1} & j_{2} & j_{3} \\
j_{4} & j_{5} & j_{3} \\
j_{6} & j_{6} & 0
\end{array}\right\}=\frac{(-1)^{j_{2}+j_{3+j_{4}+j 6}}}{\hat{j}_{3} \hat{j}_{6}}\left\{\begin{array}{lll}
j_{1} & j_{2} & j_{3} \\
j_{5} & j_{4} & j_{6}
\end{array}\right\}
$$

A 9-j with two or three zeroes reduces to one of the following cases:

$$
\begin{gathered}
\left\{\begin{array}{lll}
a & b & c \\
a & b & c \\
0 & 0 & 0
\end{array}\right\}=\frac{1}{\hat{a} \hat{b} \hat{c}} \\
\left\{\begin{array}{lll}
a & b & c \\
c & 0 & c \\
b & b & 0
\end{array}\right\}=\frac{(-1)^{a-b-c}}{\hat{b}^{2} \hat{c}^{2}} \\
\left\{\begin{array}{lll}
0 & a & a \\
a & 0 & a \\
a & a & 0
\end{array}\right\}=\frac{(-1)^{2 a}}{\hat{a}^{4}} .
\end{gathered}
$$

\section{6-j Coefficients}

The $6 \cdot j$ coefficients are invariant under any permutation of columns or against interchange of upper and lower elements in each of any two columns. A $6-j$ with a zero as one element can be reduced to

$$
\left\{\begin{array}{lll}
j_{1} & j_{2} & j_{3} \\
j_{2} & j_{1} & 0
\end{array}\right\}=\frac{(-1)^{j_{1}+j_{2}+j_{3}}}{\hat{j}_{1} \hat{j}_{2}}
$$

\section{3-j Coefficients}

The $3-j$ coefficients are invariant under an even number of permutations of columns. An odd number of permutations of columns or changing the sign of all three projection quantum numbers introduces a phase of minus one to the sum of the three angular momenta involved. The $3-j$ symbol is defined in terms of the vector-coupling coefficient as

$$
\left(\begin{array}{lll}
j_{1} & j_{2} & J \\
m_{1} & m_{2} & -M
\end{array}\right) \equiv \frac{(-1) j_{1}-j_{2}+M}{\hat{J}}\left\langle j_{1} m_{1} j_{2} m_{2} \mid J M\right\rangle
$$

and when $J=0$

$$
\left(\begin{array}{lll}
j & j & 0 \\
m-m & 0
\end{array}\right)=\frac{(-1)^{j-m}}{\hat{j}} .
$$

Phases

Often there are a number of equivalent ways of expressing a given phase factor. Some useful identities for manipulating half-integral phases follow:
(1) Phases are invariant modulo $4 j$, i.e.,

$$
(-1)^{n j}=(-1)^{(n \pm 4) j} \text {. }
$$

(2) $2 j=$ odd integer and $2\left(j_{1}+j_{2}\right)=$ even integer.

(3) Any three angular momenta which satisfy triangularity (from a $3-j, 6-j$, or $9-j$ coefficient) sum to an integer.

We always use the standard convention that $i=\sqrt{-1}$, so that $(i)^{l}=(-1)^{l / 2},(-i)^{l}=(-1)^{l}$. $(-1)^{l / 2}=(-1)^{-l / 2}$, and $(i)^{-l}=(-1)^{-l / 2}$. The reader is reminded that complex conjugation is equivalent to computing the inverse of a phase, i.e.,

$$
\left[(-1) \sum_{i}^{\sum_{i} / 2}\right]^{*}=(-1) \sum_{i}^{-l_{i} / 2} \text {. }
$$

\section{Spherical Harmonics}

Formulas involving spherical harmonics with a sum over the projection quantum numbers arise frequently in physical problems. The following are examples:

$$
\begin{gathered}
\sum_{m}\left|Y_{m}^{\lceil\eta}\right|^{2}=\sum_{m}\left|Y_{l m}\right|^{2}=\frac{2 l+1}{4 \pi} \\
\sum_{m} m\left|Y_{m}^{I l]}\right|^{2}=0 \\
\sum_{m} m^{2}\left|Y_{m}^{\lceil I \eta}\right|^{2}=\frac{l(l+1)(2 l+1)}{8 \pi} \sin ^{2} \theta \\
\sum_{m} Y_{m}^{[\eta} *(1) Y_{m}^{[\eta}(2)=\hat{l}\left[Y^{[l]}(1) \times Y^{[\ell]}(2)\right]^{[0]} \\
=\frac{2 l+1}{4 \pi} P_{l}\left(\cos \theta_{12}\right) .
\end{gathered}
$$

\section{Vectors}

On numerous occasions it is useful to express vector operations as angular momentum couplings. For example,

$$
\begin{aligned}
& \mathbf{A} \cdot \mathbf{B}=\sqrt{3}\left[A^{[1]} \times B^{[1]}\right]^{[0]} \\
& \mathbf{A} \times \mathbb{B}=\sqrt{2}\left[A^{[1]} \times B^{[1]}\right]^{[1]}
\end{aligned}
$$

and the coupling of a unit vector with a spherical harmonic to form a vector spherical harmonic:

$$
\begin{aligned}
\underset{l 1 ;, M}{[J]} & =\left[Y^{[l]} \times e^{[1]}\right]_{M}^{[J]} \\
& =\sum_{m, \lambda}\langle\operatorname{lm} 1 \lambda \mid J M\rangle Y_{m}^{[l]} e_{\lambda}^{[1]} \\
& =(-1)^{(l+1) / 2} \sum_{m \lambda}\langle\operatorname{lm} 1 \lambda \mid J M\rangle Y_{l m} e_{1 \lambda}
\end{aligned}
$$

$$
=(-1)^{(l+1) / 2} \mathscr{Y}_{J .1}^{l 1}
$$




\section{Gradient Operator}

The projection integrals for the gradient operator are

$$
\begin{aligned}
& {\left[R_{l+1} Y^{[l+1]}\left|\nabla^{[1]}\right| R_{l} Y^{[l]}\right]} \\
& \quad=(l+1)^{1 / 2}\left\langle R_{l+1}\left|\frac{\partial}{\partial r}-\frac{l}{r}\right| R_{l}\right\rangle
\end{aligned}
$$

$$
\left[R_{l-1} Y^{[l-1]}\left|\nabla^{[1]}\right| R_{l} Y^{[l]}\right]
$$

$$
=l^{1 / 2}\left\langle R_{l-1}\left|\frac{\partial}{\partial r}+\frac{l+1}{r}\right| R_{l}\right\rangle .
$$

The authors wish to express their appreciation to Michael Danos for teaching them the diagrammatic recoupling method and for a careful reading of the manuscript. John Lightbody, Jr. and James Murphy, II helped us by serving as test cases for the pedagogical aspects of this article. Finally, we thank Theresa Dunn for a fine job in preparing the manuscript.

\section{References and Notes}

[1] Danos, M., Ann. Phys. (N.Y.) 63, 319 (1971).

[2] Briggs, J. S., Rev. Mod. Phys. 43, 189 (1971).
[3] El-Baz, E., and Castel, B., Am. J. P'hys. 39, 868 (1971).

[4] Merzbacher, E., Quantum Mechanics (John Wiley \& Sons, Inc., New York, 1961).

[5] Messiah, A., Quantum Mechanics, Vol. 2. (John Wiley \& Sons, Inc., New York, 1961).

[6] Schiff, L. I., Quantum Mechanics, 3rd ed. (McGraw-Hill, New York, 1968).

[7] Edmonds, A. R., Angular Momentum in Quantum Mechanics (Princeton University Press, Princeton, N.J., 1960).

[8] O'Connell, J. S., Electromagnetic Multipole Transitions in the Recoupling Picture, Mat. Bur. Stand. (U.S.), Tech. Note 713, 23 pages (1972).

[9] Goldfarb, L. J. B., Nuclear Reactions I, edited by P. M. Endt and Demeur, M. (North-Holland Publishing Co., Amsterdam, 1959), pp. 159-214.

[10] Hayward, E., Photonuclear Reactions, Nat. Bur. Stand. (U.S.), Monogr. 118, 47 pages (1970).

[11] Källèn, G., Elementary Particle Physics (Addison-Wesley Publishing Co., Inc., USA, 1964), pp. 152-156.

[12] Fano, U., and Racah, G., Irreducible Tensorial Sets (Academic Press Inc., Publishers, N.Y., 1959), p. 23.

[13] In Danos' paper, conjugation is defined as $\Psi_{M}^{[J]^{C}}=(-1)^{J-M}$ $\Psi_{-M}^{[J]}$. Danos now suggests that eq $(\mathrm{A}-2)$ be used for conformity with Wigner and Fano-Racah.

[14] The coefficient $(-1)^{J_{2}+K+J_{2}}\left(\begin{array}{ccc}J_{1} & K & J_{2} \\ -M_{1} & m & M_{2}\end{array}\right)$ is the $\bar{V}$-coeffcient, $\bar{V}\left(\begin{array}{rrr}J_{1} & K & J_{2} \\ -M_{1} & m & M_{2}\end{array}\right)$, of Fano-Racah. Note that it arises naturally in this discussion.

[15] Note that $\chi_{\mu}^{[1 / 2]^{\dagger}} \equiv \tilde{\chi}_{\mu}^{[1 / 2]^{C}}=\chi_{\mu}^{[1 / 2]^{T}}, \chi_{\mu}^{[1 / 2]^{C}}=U \chi_{\mu}^{[1 / 2]}=(-1)^{1 / 2+\mu}$ $\chi_{-\mu}^{[1 / 2]}, \quad \tilde{\chi}_{\mu}^{[1 / 2]^{C}}=\tilde{\chi}_{\mu}^{[1 / 2]} U^{T}$ and $\bar{\chi}_{\mu}^{[1 / 2]}=-\left(\chi_{\mu}^{[1 / 2]^{c}}\right)^{T}$, where $T$ means ordinary matrix transpose. 
FORM NPS-114A (1-71)

\begin{tabular}{|c|c|c|c|c|}
\hline $\begin{array}{l}\text { U.S. DEPT. OF COMM. } \\
\text { BIBLIOGRAP:HIC DATA } \\
\text { SHEET }\end{array}$ & $\begin{array}{l}\text { 1. PUBLICATION OR REPORT NO. } \\
\text { NBS-MONOGR } 140\end{array}$ & $\begin{array}{l}\text { 2. Gov't Accession } \\
\text { No. }\end{array}$ & \multicolumn{2}{|c|}{ 3. Recipient's Accession No. } \\
\hline \multirow{2}{*}{\multicolumn{3}{|c|}{$\begin{array}{l}\text { 4. TITLE A:ND SUBTITLE } \\
\text { Graphica1 Recoupling of Angular Momenta }\end{array}$}} & \multicolumn{2}{|c|}{$\begin{array}{l}\text { 5. Publication Date } \\
\text { October } 1973\end{array}$} \\
\hline & & & \multicolumn{2}{|c|}{ 6. Performing Organization Code } \\
\hline 7. AUTHOR(S) D. R. & \multicolumn{2}{|c|}{ D. R. Lehman and J. S. O'Connell } & \multicolumn{2}{|c|}{ 8. Petforming Organization } \\
\hline \multicolumn{3}{|c|}{$\begin{array}{l}\text { 9. PERFORMING ORGANIZATION NAME AND ADDRESS } \\
\text { NATIONAL BUREAU OF STANDARDS } \\
\text { DEPARTMENT OF COMMERCE } \\
\text { WASHINGTON, D.C. } 20234\end{array}$} & \multicolumn{2}{|c|}{$\begin{array}{l}\text { 10. Project/Task/Work Unit No. } \\
2400104 \text { and } 2410231 \\
\text { 11. Contract/Grant No. }\end{array}$} \\
\hline \multirow{2}{*}{\multicolumn{3}{|c|}{$\begin{array}{l}\text { 12. Sponsoring Organization Name and Address } \\
\text { Center for Radiation Research } \\
\text { RadP, B102 }\end{array}$}} & \multicolumn{2}{|c|}{$\begin{array}{l}\text { 13. Type of Report \& Period } \\
\text { Covered } \\
\text { Final }\end{array}$} \\
\hline & & & \multicolumn{2}{|c|}{ 14. Sponsoring Agency Code } \\
\hline \multicolumn{5}{|c|}{ 15. SUP PLEMENT ARY NOTES } \\
\hline \multicolumn{5}{|c|}{$\begin{array}{l}\text { 16. ABSTRACT (A 200-word or less factual summary of most significant information. If document includes a significant } \\
\text { bibliography or literature survey, mention it here.) } \\
\qquad \text { A diagrammatic method for solving angular momentum recoupling } \\
\text { problems is presented. It is shown that a few graphical elements with a } \\
\text { set of rules for their use lead to the solution of many types of recoupling } \\
\text { problems in an intuitive and systematic way. Several examples are given } \\
\text { together with exercises to develop the reader's facility with the method. }\end{array}$} \\
\hline \multicolumn{5}{|c|}{$\begin{array}{l}\text { 17. KEY WORDS (Alphabetical order, separated by semicolons) } \\
\text { Angular momentum; diagrams; graphs; quantum theory; recoupling; } \\
\text { transformation theory. }\end{array}$} \\
\hline \multirow{2}{*}{\multicolumn{2}{|c|}{$\begin{array}{l}\text { 18. AVAILABILITY STATEMENT } \\
{[\mathrm{X} \text { UNLIMIT ED. }} \\
\square \text { FOR OFFICIAL DISTRIBUTION. DO NOT RELEASE } \\
\text { TO NTIS. }\end{array}$}} & $\begin{array}{l}\text { 19. SECURI' } \\
(\text { THIS RI } \\
\\
\text { UNCL AS }\end{array}$ & $\begin{array}{l}\text { CLASS } \\
\text { ORT) } \\
\text { IFIED }\end{array}$ & $\begin{array}{l}\text { 21. NO. OF PAGES } \\
18\end{array}$ \\
\hline & & $\begin{array}{l}\text { 20. SECURI } \\
\text { (THIS P } \\
\text { UNCL AS }\end{array}$ & $\begin{array}{l}\text { Y CLASS } \\
\text { GE) } \\
\text { IFIED }\end{array}$ & $\begin{array}{r}\text { 22. Price } \\
50 \text { cents }\end{array}$ \\
\hline
\end{tabular}



PERIODICALS

JOURNAL OF RESEARCH reports National Bureau of Standards research and development in physics, mathematics, and chemistry. Comprehensive scientific papers give complete details of the work, including laboratory data, experimental procedures, and theoretical and mathematical analyses. Illustrated with photographs, drawings, and charts. Includes listings of other NBS papers as issued.

Published in two sections, available separately:

\section{- Physics and Chemistry (Section A)}

Papers of interest primarily to scientists working in these fields. This section covers a broad range of physical and chemical research, with major emphasis on standards of physical measurement, fundamental constants, and properties of matter. Issued six times a year. Annual subscription: Domestic, $\$ 17.00$; Foreign, \$21.25.

\section{- Mathematical Sciences (Section B)}

Studies and compilations designed mainly for the mathematician and theoretical physicist. Topics in mathematical statistics, theory of experiment design, numerical analysis, theoretical physics and chemistry, logical design and programming of computers and computer systems. Short numerical tables. Issued quarterly. Annual subscription: Domestic, $\$ 9.00$; Foreign, $\$ 11.25$.

\section{DIMENSIONS, NBS}

The best single source of information concerning the Bureau's measurement, research, developmental, cooperative, and publication activities, this monthly publication is designed for the layman and also for the industry-oriented individual whose daily work involves intimate contact with science and technology - for engineers, chemists, physicists, research managers, product-development managers, and company executives. Annual subscription: Domestic, $\$ 6.50$; Foreign, $\$ 8.25$.

\section{MONPERIODICALS}

Applied Mathematics Series. Mathematical tables, manuals, and studies.

Building Science Series. Research results, test methods, and performance criteria of building materials, components, systems, and structures.

Handbooks. Recommended codes of engineering and industrial practice (including safety codes) developed in cooperation with interested industries, professional organizations, and regulatory bodies.

Special Publications. Proceedings of NBS conferences, bibliographies, annual reports, wall charts, pamphlets, etc.

Monographs. Major contributions to the technical literature on various subjects related to the Bureau's scientific and technical activities.

National Standard Reference Data Series. NSRDS provides quantitative data on the physical and chemical properties of materials, compiled from the world's literature and critically evaluated.

Product Standards. Provide requirements for sizes, types, quality, and methods for testing various industrial products. These standards are developed cooperatively with interested Government and industry groups and provide the basis for common understanding of product characteristics for both buyers and sellers. Their use is voluntary.

Technical Notes. This series consists of communications and reports (covering both other-agency and NBS-sponsored work) of limited or transitory interest.

Federal Information Processing Standards Publications. This series is the official publication within the Federal Government for information on standards adopted and promulgated under the Public Law 89-306, and Bureau of the Budget Circular A-86 entitled, Standardization of Data Elements and Codes in Data Systems.

Consumer Information Series. Practical information, based on NBS research and experience, covering areas of interest to the consumer. Easily understandable language and illustrations provide useful background knowledge for shopping in today's technological marketplace.

\section{BIBLIOGRAPHIC SUBSCRIPTION SERVICES}

The following current-awareness and literature-survey bibliographies are issued periodically by the Bureau :

Cryogenic Data Center Current Awareness Service (Publications and Reports of Interest in Cryogenics). A literature survey issued weekly. Annual subscription: Domestic, $\$ 20.00$; foreign, $\$ 25.00$.

Liquefied Natural Gas. A literature survey issued quarterly. Annual subscription: $\$ 20.00$.

Superconducting Devices and Materials. A literature survey issued quarterly. Annual subscription: $\$ 20.00$. Send subscription orders and remittances for the preceding bibliographic services to the U.S. Department of Commerce, National Technical Information Service, Springfield, Va. 22151.

Electromagnetic Metrology Current Awareness Service (Abstracts of Selected Articles on Measurement Techniques and Standards of Electromagnetic Quantities from D-C to Millimeter-Wave Frequencies). Issued monthly. Annual subscription: $\$ 100.00$ (Special rates for multi-subscriptions). Send subscription order and remittance to the Electromagnetic Metrology Information Center, Electromagnetics Division, National Bureau of Standards, Boulder, Colo. 80302.

Order NBS publications (except Bibliographic Subscription Services) from: Superintendent of Documents, Government Printing Office, Washington, D.C. 20402. 
USS. DEPARTMENT OF COMMERCE

National Bureau of Standards

Washington, D.C. 20234

OFFICIAL BUSINESS

POSTAGE AND FEES PAID

USS. DEPARTMENT OF COMMERCE

Penalty for Private Use, $\$ 300$

U.S.MAIL 